\title{
Bottom-Up/Top-Down Image Parsing with Attribute Grammar
}

\author{
Feng Han and Song-Chun Zhu
}

\begin{abstract}
This paper presents a simple attribute graph grammar as a generative representation for man-made scenes such as buildings, hallways, kitchens, and living rooms and studies an effective top-down/bottom-up inference algorithm for parsing images in the process of maximizing a Bayesian posterior probability or equivalently minimizing a description length (MDL). This simple grammar has one class of primitives as its terminal nodes, i.e., the projection of planar rectangles in 3-space into the image plane, and six production rules for the spatial layout of the rectangular surfaces. All of the terminal and nonterminal nodes in the grammar are described by attributes for their geometric properties and image appearance. Each production rule is associated with some equations that constrain the attributes of a parent node and those of its children. Given an input image, the inference algorithm computes (or constructs) a parse graph, which includes a parse tree for the hierarchical decomposition and a number of spatial constraints. In the inference algorithm, the bottom-up step detects an excessive number of rectangles as weighted candidates, which are sorted in a certain order and activate top-down predictions of occluded or missing components through the grammar rules. The whole procedure is, in spirit, similar to the data-driven Markov chain Monte Carlo paradigm [39], [33], except that a greedy algorithm is adopted for simplicity. In the experiment, we show that the grammar and top-down inference can largely improve the performance of bottom-up detection.
\end{abstract}

Index Terms-Attribute graph grammar, bottom-up/top-down, image parsing, primal sketch, generative model.

\section{INTRODUCTION}

$I^{\prime}$ $\mathrm{N}$ real-world images, especially man-made scenes such as Luildings, offices, and living spaces, a large number of visual patterns and objects can be decomposed hierarchically into a small number of primitives arranged by a small set of spatial relations. This is similar to language, where a huge set of sentences can be generated from a relatively small vocabulary through some grammar rules that group words into phrases, clauses, and sentences. In this paper, we present a simple attribute graph grammar as a generative image representation and study an effective top-down/bottom-up inference algorithm for parsing images in the process of maximizing a Bayesian posterior probability or equivalently minimizing a description length (MDL).

In the following, we shall briefly introduce the representation and algorithm and then discuss the literature and our contributions.

\subsection{Overview of the Generative Representation}

Our simple grammar has one root node for the scene, one recursive nonterminal node for objects or surfaces, one class of primitives as its terminal nodes, i.e., planar rectangular surfaces projected on images. All of the terminal and nonterminal nodes are described by attributes for their geometric properties and image appearance.

- F. Han is with Sarnoff Corporation, 201 Washington Road, Princeton, NJ 08543.E-mail: hanf@cs.ucla.edu.

- S.-C. Zhu is with the Departments of Computer Science and Statistics, 8125 Math Science Building, Box 951554, University of California, Los Angeles, Los Angeles, CA 90095. E-mail: sczhu@stat.ucla.edu.

Manuscript received 15 Apr. 2006; revised 23 Apr. 2007; accepted 2 Jan. 2008; published online 7 Mar. 2008.

Recommended for acceptance by M. Hebert.

For information on obtaining reprints of this article, please send e-mail to: tpami@computer.org, and reference IEEECS Log Number TPAMI-0296-0406. Digital Object Identifier no. 10.1109/TPAMI.2008.55.
The grammar has six production rules for the spatial layout of the rectangular surfaces. Of the six rules, one expands the root node (scene) into $m$ independent objects and one instantiates a nonterminal node to a primitive. The other four rules arrange the objects or surfaces recursively in four possible ways:

1. aligning $m$ objects in a line, for example, a row of windows in a wall,

2. nesting one object inside the other, such as a window frame,

3. aligning three rectangular surfaces in a cube, and

4. arranging up to an $m \times n$ object in a mesh/tile structure such as a tile floor.

Each production rule is associated with some equations that constrain the attributes of a parent node and those of its children.

Given an input image, our objective is to compute a hierarchical parse graph where each nonterminal node corresponds to a production rule. In this parse graph, the vertical links show the decomposition of the scene and objects into their components and the horizontal (dashed) links specify the spatial relations between components through constraints on their attributes. Fig. 1 illustrates the hierarchical representation for a kitchen scene and the computational algorithm for constructing the parse graph in an iterative bottom-up/top-down procedure.

Note that the parse graph is not predetermined but is constructed "on the fly" from the input image. It is also a generative representation instead of a discriminative model. The parse graph produces a planar configuration in the image plane. The configuration consists of rectangular line segments. These line segments are further broken into smaller image primitives for edge elements, bars, and 


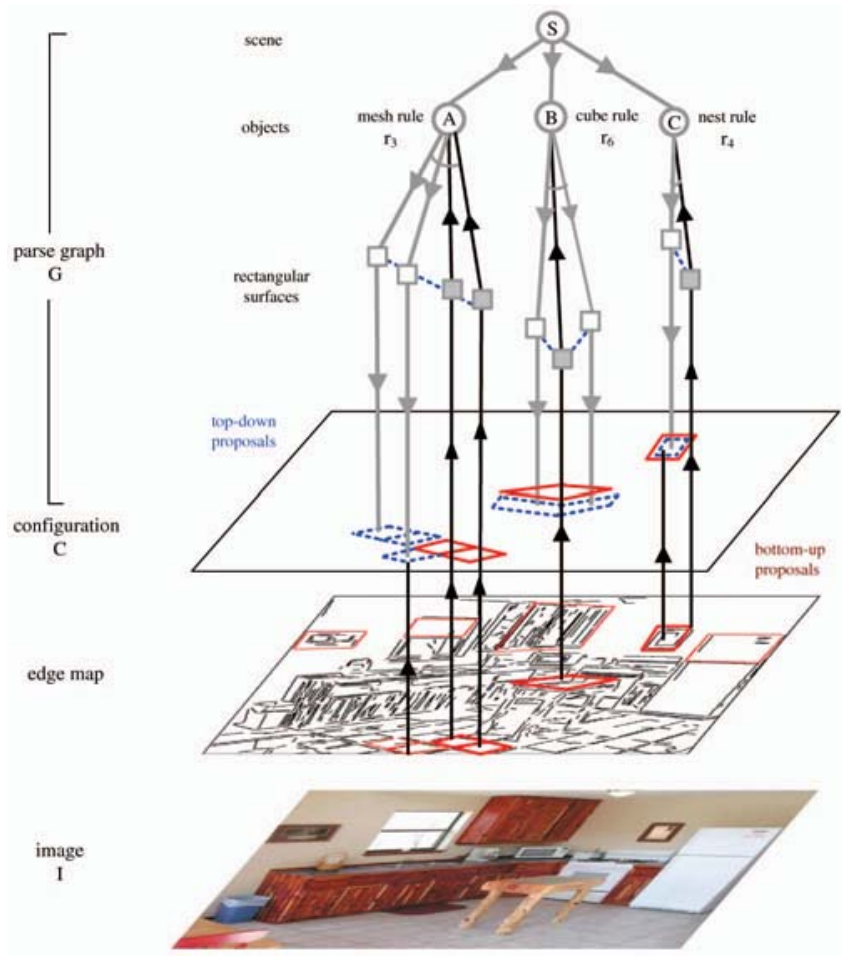

Fig. 1. The hierarchic parse graph is a generative representation, and it produces a configuration in the image plane. This configuration generates the image in a primal sketch model. The parse graph is constructed by an iterative top-down/bottom-up algorithm. The rectangular primitives detected in the bottom-up step activate the grammar rules for predicting missing or occluded components in the top-down process.

corners in an image primitive dictionary, which, in turn, generate the image by the primal sketch model [9], [10]. Therefore, our model (combined with a primal sketch model) is fully generative from the scene node to the pixels. This property enables a Bayesian formulation with a prior probability on the parse graph and a likelihood model for the primal sketch (from image primitives to pixels).

\subsection{Overview of the Top-Down/Bottom-Up Inference Algorithm}

This paper is focused on designing an effective inference algorithm that integrates top-down and bottom-up inference for attribute grammars. We adopt a greedy algorithm for maximizing the Bayesian posterior probability that proceeds in three phases.

Phase 1 is bottom-up detection. We compute edge segments from the input image and estimate a number of vanishing points (usually three) in the image by using the method studied in [38]. Then, the line segments converging to the same vanishing point are put in a line set. The rectangle hypotheses are generated in a method similar to RANSAC [7]. We draw two pairs of line segments from two out of the three line sets and then evaluate them by the goodness of fit (compatibility) to a rectangle. The two pairs of line segments that pass a minimum compatibility test become a weighted hypothesis. We thus generate an excessive number of rectangles as bottom-up proposals, which may overlap or conflict with each other and are sorted in decreasing order by their weights.
Phase 2 initializes the terminal nodes of the parse graph in a greedy way. In each step, the algorithm picks the most promising bottom-up rectangle hypothesis with the heaviest weight among all of the candidates and accepts it if it increases the Bayesian probability or reduces the description length. Then, the weights of all of the candidates that overlap or conflict with this accepted rectangle are reduced as in the matching pursuit algorithm [20].

Phase 3 integrates top-down/bottom-up inference. Each rectangle in the current parse graph matches (often partially) to a production rule with attributes passed to the nonterminal node. These nonterminal nodes are, in turn, matched to other production rules, which then generate topdown proposals for predictions (see the downward arrows in Fig. 1). The weights of the top-down proposals are calculated based on the posterior probabilities. For example, two adjacent rectangles may activate the line rule (or a mesh rule or a cube rule), which then generates a number of rectangles along the aligned axis. Some of these top-down proposals may have existed in the candidate sets of the bottom-up proposal. Such proposals bear both the upward and downward arrows and their weights increase.

In phase 3, each of the five grammar rules (omitting the scene rule) maintains a data structure that stores all of its weighted candidates. Each step of the algorithm picks the most promising proposal (with the heaviest weight) among all five candidate sets. This proposal is accepted if it increases the Bayesian probability or reduces the description length. Thus, a new nonterminal node is added to the parse graph. This corresponds to recognizing a new subconfiguration and activates the following actions: 1) creating potentially new "top-down" proposals and inserting them into the lists, 2) reweighting some proposals in the candidate sets, and 3) passing attributes between a node and its parent through the constraint equations associated with this production rule.

The top-down and bottom-up computing is illustrated in Fig. 1 for the kitchen scene. For most images, the parse graph has about three layers with about 20 nodes, so the computation can be done by AI search algorithms such as best first search. In our experiments, we observed that the top-down and prior information helps in detecting some weak rectangles that are missing in the bottom-up detection. Some "illusory rectangles" could also be hallucinated, especially due to the line and mesh grammar rules. Comparison experiments show that the top-down process improves the performance by a large margin.

\subsection{Related Work on Attribute Grammar, Rectangle Detection, and Image Parsing}

In the literature, the study of syntactic pattern recognition was pioneered by $\mathrm{Fu}$ and You [8], [36], [37], Hanson and Riseman [11], and Ohta et al. [25], [24] and other image understanding systems with top-down/bottom-up inference [2], [13], [14], [6], [21], [22] in the 1970-1980s. Its applicability has been limited by two difficulties. The first is known as the "semantic gap." The primitive patterns (terminators) used in their grammar could not be computed reliably from real images. The second is the lack of expressive power of the early work, which was mostly focused on string grammars and stochastic context-free 
grammars (SCFGs). In recent years, attribute grammars [1] and context sensitive graph grammars [29] have been developed in visual diagrams parsing. In the vision literature, grammars are mostly studied in binary shape recognition, such as the grammars for medial axis [40] and shock graphs [30]. Most recently, there has been a resurgence of compositional computing for segmentation [31], [34] and object recognition [16], [3]. However, a more general representation and computational framework has yet been developed. A comprehensive survey of these work is referred to in [42].

Detecting rectangular structures in images has been well studied in the vision literature, especially for detecting building roofs in aerial images. One class of methods [17], [19], [32] detects edge segments and line primitives and then groups them into rectangles. The other types of methods [43], [38] use Hough Transforms on edge maps to detect rectangles globally. A Markov chain Monte Carlo method was developed in rectangular scene construction in [4], which also uses compositional structures. Putting detecting rectangle structures in the broader topic of modeling structural variability, our work is also closely related to a variety of representations, including shape grammars with algebraic constraints [26], [27], [18].

Our work is also related to some previous work on object recognition [5], [15] and image parsing by data-driven Markov chain Monte Carlo (DDMCMC) [39], [33]. The common goal is to design effective algorithms by integrating bottom-up and top-down steps for inferring single objects or hierarchical image structures. In DDMCMC, each step is made reversible for backtracking and observes the detailed balance equations. Each step chooses a proposal with a certain probability and accepts the proposal with a probability. This is often computationally expensive. When the proposal is strong, especially at the early stage of computation as the proposals are sorted in decreasing order, it is often accepted with probability 1 . The reversible moves are mostly needed at places where the image is ambiguous. Thus, we adopt a greedy algorithm in this paper and accept the proposal deterministically when it increases the posterior probability.

In comparison to the previous work, this paper has the following novel aspects:

1. It extends the representation in image parsing [39], [33] with an attribute grammar, which sets the ground for recognizing generic objects with structural variabilities.

2. It derives a generative model, which is tightly integrated with the primal sketch models [10] to yield a full generative representation from a scene to pixels.

3. It develops the bottom-up and top-down mechanism for grammar-based image parsing. This strategy has been used in some recent object recognition work [3], [35].

The remainder of this paper is organized as follows: We first present the attribute grammar representation in Section 2. Then, we derive the probability models and pose the problem as Bayesian inference in Section 3. The topdown/bottom-up inference algorithm is presented in
Section 4. Some experimental results are shown in Section 5. We then conclude this paper with a discussion of future work in Section 6.

\section{Attribute Graph Grammar for Scene REPRESENTATION}

In this section, we introduce the attribute graph grammar representation to set the background for the probabilistic models in the next section.

\subsection{Attribute Graph Grammar}

An attribute graph grammar is augmented from the SCFG by including attributes and constraints on the nodes.

Definition 1. An attribute graph grammar is specified by a 5-tuple:

$$
\mathcal{G}=\left(V_{N}, V_{T}, S, \mathcal{R}, P\right),
$$

where $V_{N}$ and $V_{T}$ are the sets of nonterminal and terminal nodes, respectively, and $S$ is the initial node for the scene. $\mathcal{R}$ is a set of production rules for spatial relationships. $P$ is the probability for the grammar.

A nonterminal node is denoted by capital letters, $A, A_{1}, A_{2} \in V_{N}$, and a terminal node is denoted by lowercase letters, $a, b, c, a_{1}, a_{2} \in V_{T}$. Both nonterminal and terminal nodes have a vector of attributes denoted by $X(A)$ and $x(a)$, respectively. $\mathcal{R}=\left\{r_{1}, r_{2}, \ldots, r_{m}\right\}$ is a set of production rules expanding a nonterminal node into a number of nodes in $V_{N} \cup V_{T}$. Each rule is associated with a number of constraint equations. For example, the following is a rule that expands one node $A$ into two nodes $A_{1}, A_{2} \in V_{N}$ :

$$
r: A \rightarrow\left(A_{1}, A_{2}\right) \text {. }
$$

The associated equations are constraints on the attributes:

$$
g_{i}(X(A))=f_{i}\left(X\left(A_{1}\right), X\left(A_{2}\right)\right), i=1,2, \ldots, n(r) .
$$

$g_{i}()$ and $f_{i}()$ are usually projection functions that take some elements from the attribute vectors. For instance, let $X(A)=$ $\left(X_{1}, X_{2}, X_{3}\right)$ and let $X\left(A_{1}\right)=\left(X_{11}, X_{12}\right)$. Then, an equation could simply be an equivalence constraint (or assignment) for passing the information between nodes $A$ and $A_{1}$ in either direction, $X_{1}=X_{11}$. In the parsing process, sometimes we know the attributes of a child node $X_{11}$ and then pass it to $X_{1}$ in rule $r$. This is called "bottom-up message passing." Then, $X_{1}$ may be passed to another child node's attribute $X_{2}$, with $X_{21}=X_{1}$. This is called "top-down message passing."

A production rule may instantiate a nonterminal node to a terminal node:

$$
r: A \rightarrow a
$$

with constraints

$$
g_{i}(X(A))=f_{i}(x(a)), \quad i=1,2, \ldots, n(r) .
$$

Definition 2. A parse graph $\mathbf{G}$ is a tree-structured representation expanded from a root node $S$ by a sequence of production rules $\left(\gamma_{1}, \gamma_{2}, \ldots, \gamma_{k}\right)$ and augmented with spatial relations and constraints. 


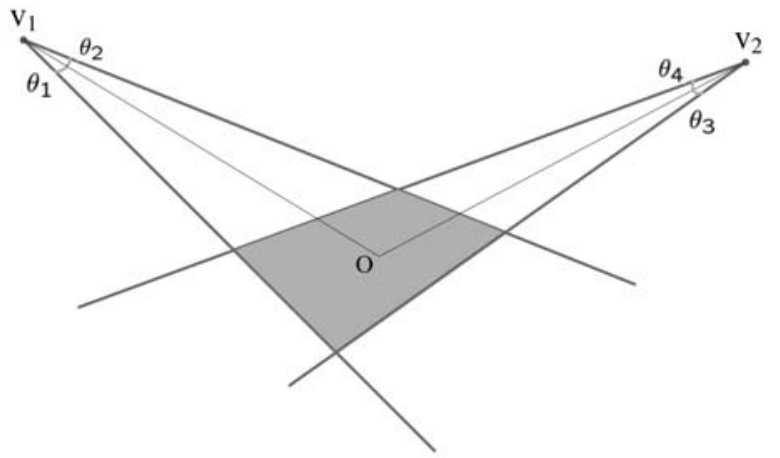

Fig. 2. A planar rectangle (shaded) is described by eight variables: the two vanishing points $v_{1}=\left(x_{1}, y_{1}\right)$ and $v_{2}=\left(x_{2}, y_{2}\right)$ and the four directions $\theta_{1}, \theta_{2}, \theta_{3}$, and $\theta_{4}$ at the two vanishing points.

Definition 3. A configuration $C$ is a set of terminal nodes (rectangles in this paper):

$$
C=\left\{\left(a_{i}, x\left(a_{i}\right)\right): a_{i} \in V_{T}, i=1,2, \ldots, K\right\} .
$$

It is deterministically generated by a parse graph, i.e., $C=C($ Graph $)$, and its attributes are denoted by $X(C)$.

If a configuration $C$ has multiple parse graph, then the grammar is said to be ambiguous.

Definition 4. A language of grammar $\mathcal{G}$ is the set of all valid configurations that can be derived by the production rules starting from a root node $S$. It is denoted by

$$
\Sigma(\mathcal{G})=\left\{(C, X(C)): S \stackrel{\gamma_{1}, \ldots, \gamma_{k}}{\longrightarrow} C, \gamma_{i} \in \mathcal{R}, i=1,2, \ldots, k\right\} .
$$

\subsection{A Class of Primitives: Rectangles}

Our simple grammar uses only one class of primitives, i.e., the projection of planar rectangles in 3-space into the image plane. As illustrated in Fig. 2, it has two pairs of parallel line segments in $3 \mathrm{D}$, which intersect at two vanishing points $v_{1}, v_{2}$ in the image plane. Therefore, the set of terminal nodes is denoted by

$$
V_{T}=\left\{(a, x(a)): x(a) \in \Omega_{a}\right\} .
$$

There are many equivalent ways of defining the attributes $x(a)$ for a rectangle. We choose the variables to simplify the constraint equations and thus denote $a$ by eight variables: two vanishing points $v_{1}=\left(x_{1}, y_{1}\right)$ and $v_{2}=\left(x_{2}, y_{2}\right)$, two orientations $\theta_{1}$ and $\theta_{2}$ for the two boundaries converging at $v_{1}$, and two orientations $\theta_{3}$ and $\theta_{4}$ for the two boundaries converging at $v_{2}$ :

$$
x(a)=\left(x_{1}, y_{1}, x_{2}, y_{2}, \theta_{1}, \theta_{2}, \theta_{3}, \theta_{4}\right) .
$$

\subsection{Six Production Rules}

As a generic grammar for image interpretation, our representation has the root node $S$ for the scene and one nonterminal node $A$ for objects and surfaces:

$$
V_{N}=\left\{(S, X(S)),(A, X(A)): X(S)=n, X(A) \in \Omega_{A}\right\} .
$$

The scene node $S$ generates $n$ independent objects. The object node $A$ can be instantiated (assigned) to a rectangle (rule $r_{5}$ ) or can be used recursively by the other four production rules: $r_{2}$ - the line production rule, $r_{3}$ - the mesh production rule, $r_{4}$-the nesting production rule, and $r_{6}$-the cube production rule. The six production rules are summarized in Fig. 3.

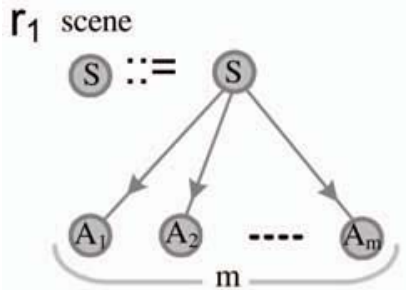

$r_{2}$ line

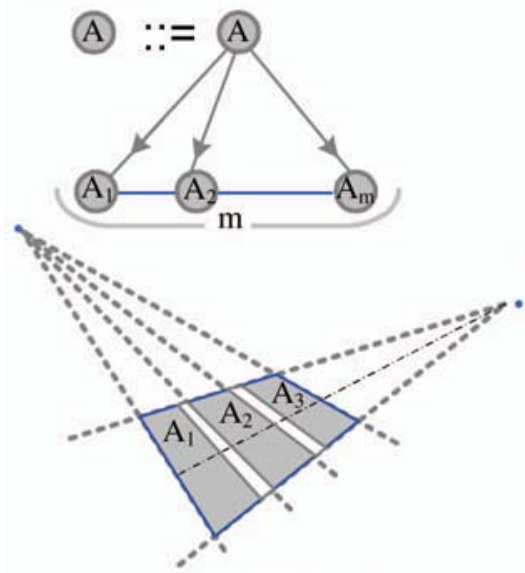

line production rule $r_{3}$ mesh

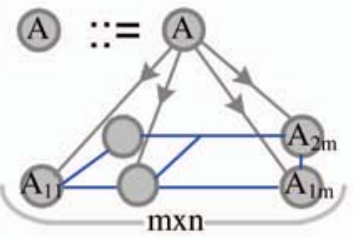

$r_{4}$ nesting
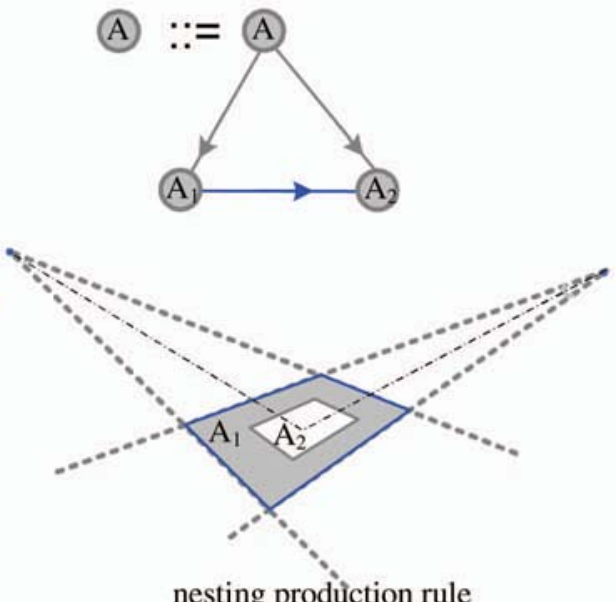

nesting prodừction rule $r_{5}$ instance

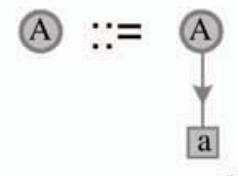

rectangle

$r_{6}$ cube
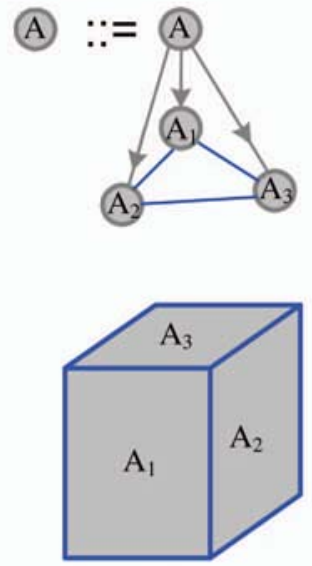

cube production rule

Fig. 3. Six attribute grammar rules. Attributes will be passed between a node to its children, and the horizontal lines show constraints on attributes. See text for explanation. 


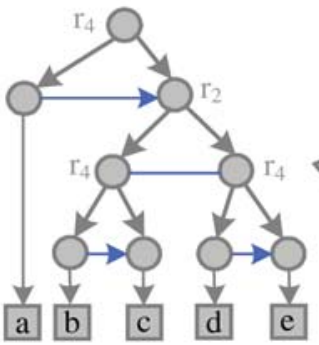

(a)

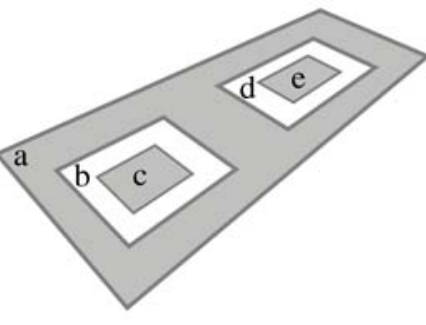

(b)

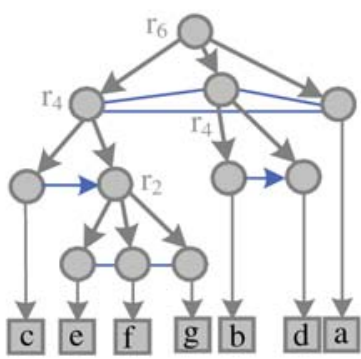

(c)

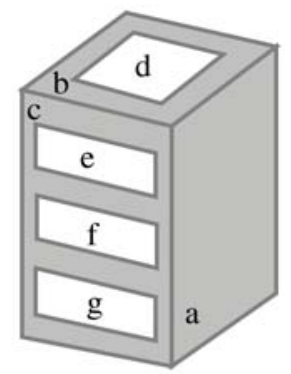

(d)

Fig. 4. (b) and (d) Two examples of rectangle object configurations and (a) and (c) their corresponding parse graphs. The production rules are shown as nonterminal nodes.

This simple grammar can generate a language with a huge number of configurations for generic objects and scenes. Fig. 4 shows two typical configurations, namely, a floor pattern and a toolbox pattern, and their corresponding parse graphs.

The attribute $X(A)=\left(\ell(A), n(A), X_{o}(A)\right)$ includes a label $\ell$ for the type of object (structure) represented by $A$, $\ell(A) \in \Omega_{\ell}=\{$ line, mesh, nest, rect, cube $\}, n(A)$ for the number of children nodes in $A$, and $X_{o}(A)$ for its geometric properties and appearance. The variables in $X_{o}(A)$ depend on the object type of $A$; therefore, we denote the attribute space of $A$ as the union of the five different subspaces:

$$
\Omega_{A}=\Omega_{A}^{\text {line }} \cup \Omega_{A}^{\text {mesh }} \cup \Omega_{A}^{\text {nest }} \cup \Omega_{A}^{\text {rect }} \cup \Omega_{A}^{\text {cube }} .
$$

The geometric attributes for all four different objects (except rectangle) are described as follows and, for clarity, we introduce the appearance attributes (intensity) in the next section, together with the primal sketch model:

1. For a line object of $n=n(A)$ rectangles,

$$
X_{o}(A)=\left(v_{1}, v_{2}, \theta_{1}, \theta_{2}, \theta_{3}, \theta_{4}, \tau_{1}, \ldots, \tau_{2(n-1)}\right) .
$$

The first eight parameters define the bounding box for the $n$ rectangles and the other $2 n-2$ orientations are for the remaining directions of the $n$ rectangles in the object.

2. For a mesh object of up to $n(A)=m \times n$ rectangles,

$$
X_{o}(A)=\left(v_{1}, v_{2}, \theta_{1}, \ldots, \theta_{4}, \tau_{1 \cdot 1}, \ldots, \tau_{2(m-1) \cdot(n-1)}\right) .
$$

Again, the first eight parameters define the bounding box for the mesh and the rest includes $2(m-$ 1) $(n-1)$ orientations for the remaining directions specifying the individual rectangles in the object, some of which could be empty.

3. For a nest object with $n(A)=2$ rectangles, $X_{o}(A)=$ $\left(v_{1}, v_{2}, \theta_{1}, \ldots, \theta_{4}, \tau_{1}, \ldots, \tau_{4}\right)$.

4. For a cube object, $n(A)=3$ and $X_{o}(A)=$ $\left(v_{1}, v_{2}, v_{3}, \theta_{1}, \theta_{2}, \theta_{3}, \theta_{4}, \theta_{5}, \theta_{6}\right)$. It has three vanishing points and three pairs of orientation angles.

Remarks. If the rectangles are arranged regularly in the line or mesh objects, for example, equally spaced, then we can omit all of the orientations $\tau_{i}$ for defining the individual rectangles. The sharing of bounding boxes and orientations are intrinsic reasons for grouping and composition as they reduce the description length. The rectangle elements in the above could be the bounding box (hidden) for other objects to allow recursive applications of the rules. In addition to these hard constraints for passing attributes among nodes, we shall introduce probabilities to impose soft constraints on the free variables (mostly the $\tau \mathrm{s}$ ) so that the elements are nearly equally spaced.

In the following, we briefly explain the constraint equations associated with the rules. In most cases, the constraint equations are straightforward but are tedious to enumerate. Therefore, we choose to introduce the typical examples.

The simplest rule is $r_{5}$ for instantiation. It assigns a rectangle and the associated attributes to a nonterminal node $A$. Therefore, the constraint equation is simply an assignment for the eight variables:

$$
r_{5}: A \rightarrow a ; X_{o}(A)=x(a) .
$$

This assignment may go in either direction in the computation.

For the line production rule $r_{2}$, we choose $m=3$ for simplicity:

$$
\begin{aligned}
r_{2}: & A \rightarrow\left(A_{1}, A_{2}, A_{3}\right), \\
& g_{i}\left(X_{o}(A)\right)=f_{i}\left(X_{o}\left(A_{1}\right), X_{o}\left(A_{2}\right), X_{o}\left(A_{3}\right)\right), i=1,2, \ldots, k .
\end{aligned}
$$

$A$ is the bounding rectangle for $A_{1}, A_{2}$, and $A_{3}$ and shares with them the two vanishing points and four orientations. Given $X_{o}(A)$, the three rectangles $A_{1}, A_{2}$, and $A_{3}$ have only 4 degrees of freedom for the two intervals and all of the other $3 \times 8-4=20$ attributes are decided by the above attribute equations. One can derive the constraint equations for the other rules in a similar way.

\section{Probability Models and Bayesian FORMULATION}

In the generative model, an input image $\mathbf{I}$ is generated by a sketch $C_{\mathrm{sk}}$, which includes the planar configuration $C=$ $C(\mathbf{G})$ produced by parse graph $\mathbf{G}$ and some free sketches (line segments), denoted by $C_{\text {free, }}$ for other nonrectangular structures: 


$$
C_{\text {sk }}=\left(C(\mathbf{G}), C_{\text {free }}\right) .
$$

In a Bayesian framework, our objective is to maximize a posterior probability:

$$
\mathbf{G}^{*}=\arg \max p\left(\mathbf{I} \mid C_{\text {sk }}\right) p(\mathbf{G}) p\left(C_{\text {free }}\right) .
$$

The prior model $p(\mathbf{G})$ is the fifth component in the definition of the grammar $\mathcal{G}$ in (1). $p\left(C_{\text {free }}\right)$ follows the primal sketch model. We discuss $p(\mathbf{G})$ and likelihood $p\left(\mathbf{I} \mid C_{\mathrm{sk}}\right)$ in the following two sections.

\subsection{Prior Model $p(\mathbf{G})$ for the Parse Graph}

Let $\Delta_{N}(\mathbf{G})$ and $\Delta_{T}(\mathbf{G})$ be the sets of nonterminal nodes (including the root node) and terminal nodes, respectively, in the parse graph $\mathbf{G}$. Then, a parse graph includes the following three sets of variables:

$$
\begin{array}{r}
\mathbf{G}=\left(\left\{\left(\ell(A), n(A), X_{o}(A)\right): \forall A \in \Delta_{N}(\mathbf{G})\right\},\right. \\
\left\{X_{o}(a): \forall a \in \Delta_{T}(\mathbf{G})\right) .
\end{array}
$$

Due to the hierarchical tree structure, we factorize the probability $p(\mathbf{G})$ as

$$
\begin{aligned}
p(\mathbf{G})= & \prod_{A \in \Delta_{N}(\mathbf{G})}\left[p(\ell(A)) p(n(A) \mid \ell(A)) p\left(X_{o}(A) \mid \ell(A), n(A)\right)\right. \\
& \left.\prod_{B \in \operatorname{child}(A)} p\left(X_{o}(B) \mid X_{o}(A)\right)\right] .
\end{aligned}
$$

First, $\ell(A)$ is a "switch" variable for selecting one of the rules. We denote the probabilities for the five rules as $q(\ell)$, which sum to one: $\sum_{\ell \in \Omega_{\ell}} q(\ell)=1$.

Second, at the root $S$ and nonterminal node $A$, we have a variable $n(A)$ for the number of their children and $p(n(A) \mid \ell(A))$ penalizes this number (or complexity) such as $p(n(A) \mid \ell(A))=\frac{\beta_{\ell(A)}(A) e^{-\beta_{\ell(A)}}}{n(A) !} \cdot p(n(A) \mid \ell(A))$ is deterministic when $A$ is the cube or the nesting rule:

$$
\begin{aligned}
& p(n(A)=3 \mid \ell(A)=\text { "cube") }=1 \text { and } \\
& p(n(A) \neq 3 \mid \ell(A)=\text { "cube" })=0 ; \\
& p(n(A)=2 \mid \ell(A)=\text { "nest") }=1 \text { and } \\
& p(n(A) \neq 2 \mid \ell(A)=\text { "nest") })=0 .
\end{aligned}
$$

Third, $p\left(X_{o}(A) \mid \ell(A), n(A)\right)=\frac{1}{Z} e^{-\phi_{\ell(A)}\left(X_{o}(A)\right)}$ (the normalization constant $Z$ can be computed empirically either by sampling or integration) is a singleton probability on the geometric and the appearance of $A$. Similarly, we have $p\left(X_{o}(B) \mid X_{o}(A)=\frac{1}{\pi} e^{-\psi_{\ell}(A)\left(X_{o}(A), X_{o}(B)\right)}\right.$ for those of $A^{\prime}$ s children. The potential functions $\phi()$ and $\psi()$ take quadratic forms to enforce some regularities, such as ensuring that aligned rectangles in a group have almost the same shape and are evenly spaced. For example, the potential functions for a line rule $A \rightarrow\left(A_{1}, A_{2}, A_{3}\right)$ (with $n(A)=3$ and the aligning axis being denoted as $\uparrow$ ) are

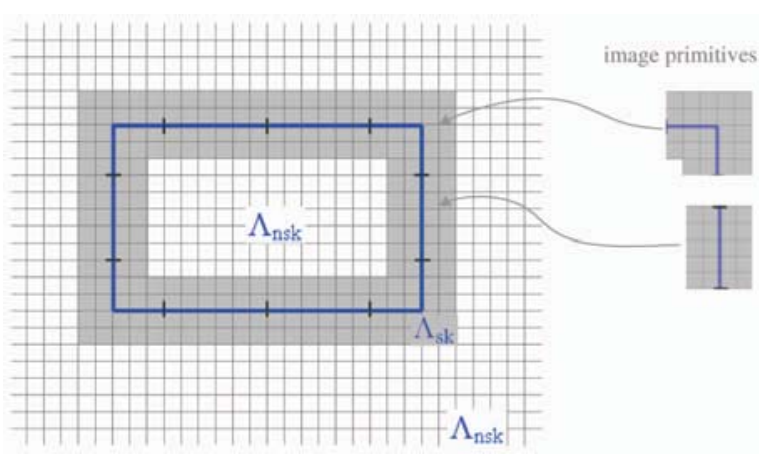

Fig. 5. Partition of image lattice $\Lambda$ into two parts: the shaded pixels $\Lambda_{\mathrm{sk}}$ around the rectangles and the remaining part $\Lambda_{\text {nsk }}$. The rectangles are divided into small edge and corner segments. Therefore, $\Lambda_{\mathrm{sk}}$ is divided into many image primitives.

$$
\begin{aligned}
& \phi_{\text {line }}\left(X_{o}(A)\right)= \sum_{i=1}^{2}\left(d\left(X_{o}\left(A_{i}\right), X_{o}\left(A_{i+1}\right)\right)-\bar{d}\right)^{2} \\
&+\frac{1}{2} \sum_{i=1}^{3}\left(w\left(X_{o}\left(A_{i}\right)\right)-\bar{w}\right)^{2}, \\
& \bar{d}= \frac{1}{2} \sum_{i=1}^{2} d\left(X_{o}\left(A_{i}\right), X_{o}\left(A_{i+1}\right)\right), \\
& \bar{w}= \frac{1}{3} \sum_{i=1}^{3} w\left(X_{o}\left(A_{i}\right)\right), \\
& \psi_{\text {line }}\left(X_{o}(A), X_{o}\left(A_{i}\right)\right)=\left(\left|\theta_{3 i}-\theta_{3}\right|^{2}+\left|\theta_{4 i}-\theta_{4}\right|^{2}\right) .
\end{aligned}
$$

In (18), $d\left(X_{o}\left(A_{i}\right), X_{o}\left(A_{j}\right)\right)$ is a function for computing the distance between the neighboring $A_{i}$ and $A_{j}$, which is defined as the minimum distance between the four boundary lines perpendicular to $\uparrow$ from $A_{i}$ and $A_{j}$, and $w\left(A_{i}\right)$ is a function for computing the distance between the two boundary lines perpendicular to $\uparrow$ from $A_{i}$. In (19), $\theta_{3}$ and $\theta_{4}$ are the two orientations of the two boundaries of $A$ parallel to $\uparrow$, while $\theta_{3 i}$ and $\theta_{4 i}$ are the two orientations of the two boundaries of $A_{i}$ parallel to $\uparrow . \phi_{\text {line }}\left(X_{o}(A)\right)$ globally constrains $A_{1}, A_{2}$, and $A_{3}$ to have similar shape and be evenly spread in the line group, while $\psi_{\text {line }}\left(X_{o}(A), X_{o}\left(A_{i}\right)\right)$ enforces the individual properties of $A_{i}$ to fit with respect to the whole group.

In our recent work [42], $p(\mathbf{G})$ is generalized to a nonfactorized form for context sensitive graph grammar.

\subsection{Likelihood Model $p\left(\mathbf{I} \mid C_{\text {sk }}\right)$}

For the likelihood model, we adopt the primal sketch model for $p\left(\mathbf{I} \mid C_{\mathrm{sk}}\right)$ and refer to two previous papers [9], [10] for this model and algorithm. The reconstruction (synthesis) of images using a configuration is shown in the experiment section (see Figs. 10 and 11). In the following, we briefly introduce the model for this paper to be self contained.

$C(\mathbf{G})$ is a set of rectangles in the image plane. Fig. 5 shows a rectangle in a lattice. A lattice is denoted by $\Lambda$ and is divided into two disjoint parts: the sketchable part for the shaded pixels around the rectangles and the nonsketchable part for the remaining part:

$$
\Lambda=\Lambda_{\mathrm{sk}} \cup \Lambda_{\mathrm{nsk}}, \quad \Lambda_{\mathrm{sk}} \cap \Lambda_{\mathrm{nsk}}=\emptyset .
$$




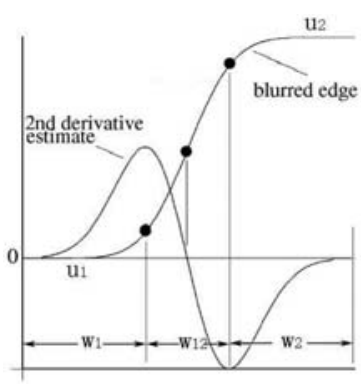

(a)

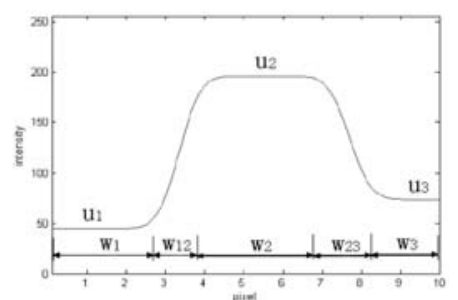

(b)
Fig. 6. The parametric representation of an edge profile and a ridge profile (from Guo et al., 2005). (a) Edge profile. (b) Ridge profile.

$\Lambda$ includes pixels that are $2 \sim 5$ pixels away from the rectangle boundaries. The rectangles are divided into short segments of 5-11 pixels long for lines and corners. Therefore, $\Lambda_{\text {sk }}$ is divided into $N$ image primitives (patches) of $5 \times 7$ pixels along these segments:

$$
\Lambda_{\mathrm{sk}}=\cup_{k=1}^{N} \Lambda_{\mathrm{sk}, \mathrm{k}} .
$$

For example, Fig. 5 shows two image primitives: one for line segment and one for a corner. The primal sketch model collects all of these primitives in a primitive dictionary represented (clustered) in parametric form:

$$
\Delta_{\mathrm{sk}}=\left\{B_{t}(u, v ; x, y, \tau, \sigma, \Theta): \forall x, y, \tau, \sigma, \theta, t\right\} .
$$

$t$ indexes the type of primitives, such as edges, bars, corners, and crosses, and $(u, v)$ are the coordinates of the patch centered at $(x, y)$ with scale $\sigma$ and orientation $\tau$. $\Theta$ denotes the parameters for the intensity profiles perpendicular to each line segment. A corner will have two profiles. The intensity profiles along the line segment in a primitive are assumed to be the same.

Therefore, there are two types of profiles, as Fig. 6 shows: One is a step edge at various scales (due to blurring effects) and the other is a ridge (bar). The step edge profile is specified with five parameters- $\Theta=\left(u_{1}, u_{2}, w_{1}, w_{12}, w_{2}\right)$ which denote the left intensity, the right intensity, the width of the left intensity (from the leftmost to the left second derivative extrema), the blurring scale, and the width of the right intensity, respectively, as shown in Fig. 5. The ridge profile is represented by seven parameters: $\Theta=$ $\left(u_{1}, u_{2}, u_{3}, w_{1}, w_{12}, w_{2}, w_{23}, w_{3}\right)$. A more detailed description is given in [10]. Using the above edge/ridge model, the onedimensional intensity function of the profile for the rectangle boundaries can be fully obtained.

Therefore, we obtain a generative model for the sketchable part of the image:

$$
\begin{gathered}
\mathbf{I}(x, y)=B_{t_{k}}\left(x-x_{k}, y-y_{k} ; \tau_{k}, \sigma_{k}, \Theta_{k}\right)+n(x, y), \\
(x, y) \in \Lambda_{\mathrm{sk}, \mathrm{k}}, \forall k=1,2, \ldots, N .
\end{gathered}
$$

The residue is assumed to be i.i.d. Gaussian noise $n(x, y) \sim G\left(0, \sigma_{o}^{2}\right)$. This model is sparser than the traditional wavelet representation as each pixel is represented by only one primitive.

As mentioned previously, rectangles are only part of the sketchable structures in the images, though they are the most common structures in man-made scenes. The remaining structures are represented as free sketches, which are object boundaries that cannot be grouped into rectangles. These free sketches are also divided into short line segments and are therefore represented by image primitives in the same way as the rectangles.

The nonsketchable part is modeled as textures without prominent structures, which are used to fill in the gaps in a way similar to image inpainting. $\Lambda_{\text {nsk }}$ is divided into a number of $M=3 \sim 5$ disjoint homogeneous texture regions by clustering the filter responses:

$$
\Lambda_{\text {nsk }}=\cup_{m=1}^{M} \Lambda_{\text {nsk,m } \mathrm{m}} .
$$

Each texture region is characterized by the histograms of some Gabor filter responses:

$$
h\left(\mathbf{I}_{\Lambda_{\text {nsk, }, \mathrm{m}}}\right)=\mathbf{h}_{m}, \quad m=1,2, \ldots, M .
$$

The probability model for the textures is the FRAME model [41], with the Lagrange parameters (vector) $\beta_{m}$ being the learned potentials. These textures use the sketchable part $\Lambda_{\mathrm{sk}}$ as the boundary condition in calculating the filter responses.

In summary, we have the following primal sketch model for the likelihood:

$$
\begin{aligned}
p\left(\mathbf{I} \mid C_{\mathrm{sk}}\right)=\frac{1}{Z} \exp \{ & -\sum_{k=1}^{N} \sum_{(x, y) \in \Lambda_{\mathrm{sk}, \mathrm{k}}} \frac{\left(\mathbf{I}(x, y)-B_{k}(x, y)\right)^{2}}{2 \sigma_{o}^{2}} \\
& \left.-\sum_{m=1}^{M}\left\langle\beta_{m}, h\left(\mathbf{I}_{\Lambda_{\mathrm{nsk}, \mathrm{m}}}\right)\right\rangle\right\} .
\end{aligned}
$$

The above likelihood is based on the concept of primitives, not rectangles. Therefore, the recognition of rectangles or larger structures (cube, mesh, etc.) only affects the likelihood locally. In other words, our parse graph is built on the primal sketch representation. This is important in designing an effective inference algorithm in the next section.

One may argue for a region-based representation by assuming homogeneous intensities within each rectangles. We find that the primal sketch has the following advantages over a region-based representation: 1) The intensity inside a rectangle can be rather complex to model as it may include shading effects, textures, and surface markings, 2) the rectangles are occluding each other (one has to infer a partial-order relation between the rectangles, i.e., a layer representation, so that the region-based model can be applied properly, and this needs extra computation), and 3) besides all of the regions covered by the rectangles, one still needs to model the background. Thus, the detection of a rectangle must be associated with fitting the likelihood for the rectangle region. In comparison, the primal sketch model largely reduces the computation.

\section{InfERENCE ALGORIthM}

Our objective is to compute a parse graph $\mathbf{G}$ by maximizing the posterior probability formulated in the previous section. The algorithm should achieve two difficult goals: 1) constructing the parse graph, whose structure is not predetermined but is constructed "on the fly" from the input 
image and primal sketch representation and 2) estimating and passing the attributes in the parse graph.

There are several ways to infer the optimal parse graph and DDMCMC has been used in [39], [33]. In this paper, our domain is limited to rectangle scenes and the parse graph is not too big (usually $\sim 20$ nodes). Thus, the best first search algorithm in AI can be directly applied to compute the parse graph by maximizing the posterior probability in the steepest ascent way. This algorithm is, in spirit, very similar to DDMCMC.

Our algorithm consists of three phases. In phase 1, we compute a primal sketch representation and initialize the configuration to the free sketches. Then, a number of rectangle proposals are generated from the sketch by a bottom-up detection algorithm. In phase 2, we adopt a simplified generative model by assuming independent rectangles (only $r_{5}$ and $r_{1}$ are considered). Thus, we recognize a number of rectangles proposed in phase 1 to initialize rule $r_{5}$ in the parse graph. The algorithm in phase 2 is very much like matching pursuit [20]. Finally, phase 3 constructs the parse graph with bottom-up/topdown mechanisms.

\subsection{Phase 1: Primal Sketch and Bottom-Up Rectangle Detection}

We start with edge detection and edge tracing to get a number of long contours. Then, we compute a primal sketch representation $C_{\mathrm{sk}}$ by using the likelihood model in (22). We segment each long contour into a number of $n$ straight line segments by polygon approximation. In manmade scenes, the majority of line segments are aligned with one of the three principal directions and each group of parallel lines intersects at a vanishing point due to perspective projection. We define all lines ending at a vanishing point to be a parallel line group. A rectangle has two pairs of parallel lines, which belong to two separate parallel line groups. We run the vanishing point estimation algorithm [38] to group all of the line segments into three groups corresponding to the principal directions. With these three line groups, we generate the rectangle hypotheses as in RANSAC [7]. We exhaustively choose two line candidates from each set, as shown in Fig. 7a, and run some simple compatibility tests on their positions to see whether two pairs of lines delineate a valid rectangle. For example, the two pairs of line segments should not intersect each other, as shown in Fig. 7b. This will eliminate some obviously inadequate hypotheses.

This yields an excessive number of bottom-up rectangle candidates, denoted by

$$
\Phi=\left\{\pi_{1}, \ldots, \pi_{L}\right\} .
$$

These candidates may conflict with each other. For example, two candidate rectangles may share two or more edge segments and only one of them should appear. We mark this conflicting relation among all of the candidates. Thus, if one candidate is accepted in the later stage, those conflicting candidates will be downgraded or eliminated.

\subsection{Phase 2: Pursuing Independent Rectangles to Initialize the Parse Graph}

The computation in phase 1 results in a free sketch configuration $C_{\text {sk }}=C_{\text {free, }} C(\mathbf{G})=\emptyset$, and a set of rectangle candidates $\Phi$. In phase 2 , we shall initialize the terminal nodes of the parse graph.

We adopt a simplified model that uses only two rules: $r_{1}$ and $r_{5}$. This model assumes that the scene consists of a number of independent rectangles selected from $\Phi$, which explains away some line segments, and the remaining lines are free sketches. A similar model has been used on signal decomposition with wavelets and sparse coding; thus, our method for selecting the rectangles is similar to the matching pursuit algorithm [20].

Rectangle Pursuit: initialize the terminal nodes of $\mathbf{G}$

Input candidate set $\Phi=\left\{\pi_{1}, \pi_{2}, \ldots, \pi_{M}\right\}$ from phase I.

1. Initialize parse graph $\mathbf{G} \leftarrow \emptyset, m=0$.

2. Compute weight $\omega_{i}$ for $\pi_{i} \in \Phi, i=1, \ldots,|\Phi|$, thus obtain

$$
\left\{\left(\pi_{i}, \omega_{i}\right): i=1,2, \ldots,|\Phi|\right\} .
$$

3. Select a rectangle $\pi_{+}$with the highest weight in $\Phi$,

$$
\omega\left(\pi_{+}\right)=\max \{\omega(\pi): \pi \in \Phi\} .
$$

4. Create a non-terminal node $A_{+}$in graph $\mathbf{G}$,

$$
\begin{aligned}
& \mathbf{G} \leftarrow \mathbf{G} \cup\left\{A_{+}\right\}, \\
& \Phi \leftarrow \Phi \backslash\left\{\pi_{+}\right\}, m \leftarrow m+1 . \\
& C(\mathbf{G}) \leftarrow C(\mathbf{G}) \cup\left\{\pi_{+}\right\} .
\end{aligned}
$$

5. Update the weights $\omega(\pi)$ for $\pi \in \Phi$ if $\pi$ overlaps with $\pi_{+}$

6. Repeat 3-5 until $\omega\left(\pi_{+}\right) \leq \delta_{0}$.

Output a set of independent rectangles $\mathbf{G}=\left\{A_{1}, A_{2}, \ldots, A_{m}\right\}$.

In the following, we calculate the weight $\omega(\pi)$ for each rectangle $\pi \in \Phi$ and the weight change.

A rectangle $\pi \in \Phi$ is represented by a number of short line segments and corners (primitives) denoted by $L(\pi)$, some of which are detected in $C_{\text {free }}$ and some of which are missing. The missing components are the missing edges or gaps between primitives in $C_{\text {free. }}$. Thus, we define two sets:

$$
L(\pi)=L_{\text {on }}(\pi) \cup L_{\text {off }}(\pi), \quad \text { with } \quad L_{\text {on }}(\pi)=L(\pi) \cap C_{\text {free }} .
$$

Suppose that, at step $m$, the current representation includes a number of rectangles in $C(\mathbf{G})$ and a free sketch $C_{\text {free: }}$ :

$$
\mathbf{G}, C_{\mathrm{sk}}=\left(C(\mathbf{G}), C_{\text {free }}\right) \text {. }
$$

Steps 3 and 4 in the above pursuit algorithm select $\pi_{+}$. Then, the new representation will be

$$
\begin{aligned}
\mathbf{G}^{\prime} & =\mathbf{G} \cup\left\{A_{+}\right\}, C\left(\mathbf{G}^{\prime}\right)=C(\mathbf{G}) \cup L\left(\pi_{+}\right), \\
C_{\text {free }}^{\prime} & =C_{\text {free }} \backslash L_{\mathrm{on}}\left(\pi_{+}\right), C_{\text {sk }}^{\prime}=\left(C\left(\mathbf{G}^{\prime}\right), C_{\text {free }}^{\prime}\right) .
\end{aligned}
$$




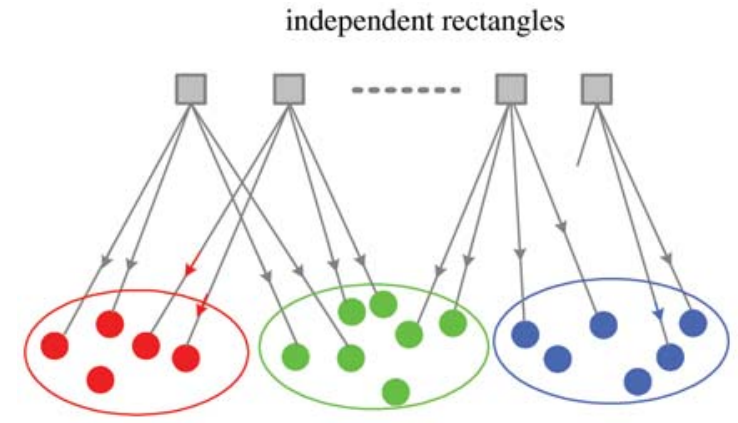

line segments grouped in three sets by vanishing points
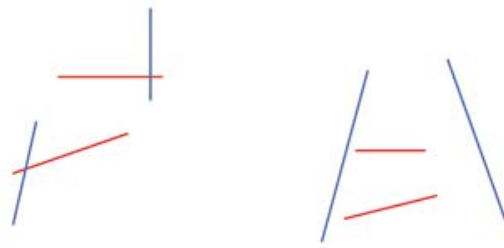

examples of incompatible hypotheses

(b)

Fig. 7. Bottom-up rectangle detection. The $n$ line segments are grouped into three sets according to their vanishing points. Each rectangle consists of two pairs of nearly parallel line segments (represented by a small circle). (a) Line segments grouped in three sets by vanishing points. (b) Examples of incompatible hypotheses.

The weight of $\pi_{+}$will be the change (or equally the logratio) of log-posterior probabilities in (15):

$$
\omega\left(b_{+}\right)=\log \left[\frac{p\left(\mathbf{I} \mid C_{\mathrm{sk}}^{\prime}\right)}{p\left(\mathbf{I} \mid C_{\mathrm{sk}}\right)} \cdot \frac{p\left(G^{\prime}\right)}{p(G)} \cdot \frac{p\left(C_{\text {free }}^{\prime}\right)}{p\left(C_{\text {free }}\right)}\right] .
$$

Choosing a rectangle $\pi_{+}$with the largest weight $\omega\left(\pi_{+}\right)>0$ increases the posterior probability in a greedy fashion. The weight can be interpreted in three terms which are computed easily. The first term, $\log \frac{p\left(\mathbf{I} \mid C_{\mathrm{sk}}^{\prime}\right)}{p\left(\mathbf{I} C_{\mathrm{kk}}\right)}$, measures the changes of the log-likelihood in a small domain covered by the primitives in $L_{\text {off }}\left(\pi_{+}\right)$. Pixels in this domain belonged to $\Lambda_{\text {nsk }}$ before and are in $\Lambda_{r m s k}$ after adding $\pi_{+}$. The likelihood does not change for any other pixels. The second term, $\log \frac{p\left(G^{\prime}\right)}{p(G)}$, penalizes the model complexity of rectangles (see $(17))$. The third term, $\log \frac{p\left(C_{\text {free }}^{\prime}\right)}{p\left(C_{\text {free }}\right)}$, awards the reduction of complexity in the free sketch.

The above weights are computed independently for each $\pi \in \Phi$. After adding $\pi_{+}$in step 5 , we should update the weight $\omega(\pi) \in \Omega$ if $\pi$ overlaps with $\pi_{+}$, i.e.,

$$
L(\pi) \cap L\left(\pi_{+}\right) \neq \emptyset,
$$

because the update of $C_{\text {free }}$ and $C(\mathbf{G})$ in step 4 changes the first and third terms in calculating $\omega(\pi)$ in (24). This update of weight involves only a local computation on $L(\pi) \cap L\left(\pi_{+}\right)$. When we detect the rectangles in phase 1 , we have computed the overlapping information. This weight update was used in wavelet pursuit, where it is interpreted as "lateral inhibition" in neuroscience.

\subsection{Phase 3: Bottom-Up and Top-Down Construction of a Parse Graph}

The algorithm for constructing the parse graph adopts a similar greedy method as in phase 2 . In phase 3 , we include the four other production rules, $r_{2}, r_{3}, r_{4}$, and $r_{6}$, and use the top-down mechanism for computing rectangles that may have been missed in the bottom-up detection. We start with an illustration of the algorithm for the kitchen scene.

In Fig. 1, the four rectangles (in red) are detected and accepted in the bottom-up phases 1 and 2. They generate a number of candidates for larger groups by using the production rules and three of these candidates are shown as nonterminal nodes $A, B$, and $C$, respectively. We denote each candidate by

$$
\Pi=\left(r_{\Pi}, A_{(1)}, \ldots, A_{\left(n_{\Pi}\right)}, B_{(1)}, \ldots, B_{\left(k_{\Pi}\right)}\right) .
$$

In the above notation, $r_{\Pi}$ is the production rule for the group. It represents a type of spatial layout or relationship of its components. For example, $A, B$, and $C$ in Fig. 1 use the mesh $r_{3}$, cube $r_{6}$, and nesting $r_{4}$ rules, respectively. In $\Pi$, $A_{(i)}, i=1,2, \ldots, n_{\Pi}$, are the existing nonterminal nodes in $\mathbf{G}$ which satisfy the constraint equations of rule $r . A_{(i)}$ can be either a nonterminal rectangle accepted by rule $r_{5}$ in phase 2 or the bounding box of a nonterminal node with three rules $r_{2}, r_{3}$, and $r_{4}$. The cube object does not have a natural bounding box. We call $A_{(i)}, i=1,2, \ldots, n_{\Pi}$, the bottom-up nodes for $\Pi$ and they are illustrated by the upward arrows in Fig. 1 . In contrast, $B_{(j)}, j=1,2, \ldots, k_{\Pi}$, are the top-down nonterminal nodes predicted by rule $r_{\Pi}$ and they are shown by the blue rectangles in Fig. 1, with downward arrows. Some of the top-down rectangles may have already existed in the candidate set $\Phi$ but have not been accepted in phase 2 or simply do not participate in the bottom-up proposal of $\Pi$. Such nodes bear both upward and downward arrows.

Fig. 8 shows the five candidate sets for the five rules. $\Psi_{i}$ is the candidate set of rule $r_{i}$ for $i=2,3,4,6$, respectively. Each candidate $\Pi \in \Psi_{i}$ is shown by an ellipse containing a number of circles $A_{(1)}, i=1, \ldots, n_{\Pi}$ (with upward arrows) and $B_{(j)}, j=1, \ldots, k_{\Pi}$ (with downward arrows). These candidates are weighted in a similar way as in the rectangles in $\Phi$ by the log-posterior probability ratio.

$$
\Psi_{i}=\left\{\left(\Pi_{j}, \omega_{j}\right): i=1,2, \ldots N_{i}\right\}, i=2,3,4,6 .
$$

$\Phi=\left\{\left(\pi_{i}, \omega_{i}\right): i=1,2, \ldots, M\right\}$ for rule $r_{5}$ has been discussed in phase 2. Now, $\Phi$ also contains top-down candidates shown by the circles with downward arrows. They are generated by other rules. A nonterminal node $A$ in graph $\mathbf{G}$ may participate in more than one group of candidate Пs, just as a line segment may be part of multiple rectangle candidates $\pi \mathrm{s}$. This creates overlaps between the candidates and needs to be resolved in a generative model.

At each step, the parsing algorithm will choose the candidate with the largest weight from the five candidate 
sets and add a new nonterminal node to the parse graph. If the candidate is $\pi \in \phi$, it means accepting a new rectangle. Otherwise, the candidate is a larger structure $\Pi$ and the algorithm creates a nonterminal node of type $r$ by grouping the existing nodes $A_{(i)}, i=1,2, \ldots, n_{\Pi}$, and inserts the topdown rectangles $B_{(j)}, j=1, \ldots, k_{\Pi}$, into the candidate set $\Phi$.

The key part of the algorithm is to generate proposals for $\pi \mathrm{s}$ and $\Pi \mathrm{s}$ and maintain the five weighted candidate sets $\Phi$, $\Psi_{i}, i=2,3,4,6$, at each step. We summarize the algorithm as follows:

\section{The algorithm for constructing the parse graph $\mathbf{G}$}

Input $\mathbf{G}=\left\{A_{1}, \ldots, A_{m}\right\}$ from phase II and $\Phi=\left\{\left(\pi_{i}, \omega_{i}\right)\right.$ :

$i=1, \ldots, M-m\}$ from phase I.

1. For rule $r_{i}, i=2,3,4,6$.

Create candidate set $\Psi_{i}=\operatorname{Proposal}\left(\mathbf{G}, r_{i}\right)$.

Compute the weight $\omega(\Pi)$ for $\Pi \in \Psi_{i}$.

2. Select a candidate with the heaviest weight, create a new

node $A_{+}$with bounding box.

$\omega_{+}\left(A_{+}\right)=\max \left\{\omega(A): A \in \Phi \cup \Psi_{2} \cup \Psi_{3} \cup \Psi_{4} \cup \Psi_{6}\right\}$.

3. Insert $A_{+}$to the parse graph $\mathbf{G} \mathbf{G} \leftarrow \mathbf{G} \cup\left\{A_{+}\right\}$.

4. Set the parent node of $A_{+}$to the non-terminal node which

proposed $A_{+}$in the top-down phase or to the root $S$ if $A_{+}$

was not proposed in top-down.

5. If $A_{+}=\pi \in \Phi$ is a single rectangle, then

Add the rectangle to the configuration: $C(\mathbf{G}) \leftarrow C(\mathbf{G}) \cup\left\{\pi_{+}\right\}$.

6. else $A_{+}=\Pi=\left(r_{\Pi}, A_{(1)}, \ldots, A_{\left(n_{\Pi}\right)}, B_{(1)}, \ldots, B_{\left(k_{\Pi}\right)}\right)$, then

Set $A_{+}$as the parent node of $A_{1(\Pi)}, \ldots, A_{n(\Pi)}$

Insert top-down candidates $B_{(1)}, \ldots, B_{\left(k_{\Pi}\right)}$ in $\Phi$ with parent

nodes $A_{+}$.

7. Augment the candidate sets $\Psi_{i}, i=2,3,4,6$ with the new node $A_{+}$.

8. Compute weights for the new candidates and update $\omega(\Pi)$

if $\Pi$ overlaps with $A_{+}$.

9. Repeat 2-8 until $\omega_{+}$is smaller than a threshold $\delta_{1}$.

Output a parse graph G.

Fig. 9 shows a snapshot of one iteration of the algorithm on the kitchen scene. Fig. $9 \mathrm{~b}$ is a subset of rectangle candidates $\Phi$ detected in phase 1 . We show a subset for clarity. At the end of phase 2, we obtain a parse graph $\mathbf{G}=\left\{A_{1}, A_{2}, \ldots, A_{21}\right\}$ whose configuration $C(\mathbf{G})$ is shown in Fig. 9c. By calling the function $\operatorname{Proposal}\left(\mathbf{G}, r_{i}\right)$, we obtain the candidate sets $\Psi_{i}, i=2,3,4,6$. The candidate sets are shown in Figs. 9d, 9e , and 9f. For each candidate, $\Pi=$ $\left.\left(r_{\Pi}, A_{(1)}, \ldots, A_{\left(n_{\Pi}\right)}, B_{(1)}, \ldots, B_{\left(k_{\Pi}\right)}\right)\right), A_{(i)}, i=1,2, \ldots, n_{\Pi}$, are shown in solid lines and $B_{(j)}, j=1,2, \ldots, k_{\pi}$, are shown in dotted lines.

The function Proposal $\left(\mathbf{G}, r_{i}\right)$ for generating candidates from the current nodes $\mathbf{G}=\left\{A_{i}: i=1,2, \ldots, m\right\}$ by using $r_{i}$ is not so hard because the set $|\mathbf{G}|$ is relatively small $(m<$ 50) in almost all examples. Each $A_{i}$ has a bounding box (except the cubes), with eight parameters for the two vanishing points and four orientations. We can simply test any two nodes $A_{i}$ and $A_{j}$ by the constraint equations of $r_{i}$. It is worth mentioning that each $A \in \mathbf{G}$ alone creates a candidate $\Pi$ for each of rules $r_{2}, r_{3}, r_{4}$, and $r_{6}$, with $n(\Pi)=1$. In such cases, the top-down proposals $B_{(j)}$, $j=1, \ldots, k_{\Pi}$, are created using both the constraint equations of $r_{i}$ and the edge maps. For example, based on one rectangle $A_{8}$, the top of the kitchen table in Fig. 9c, it proposes two rectangles by the cube rule $r_{6}$ in Fig. 9 . The parameters of those two rectangles are decided by the constraint equations of $r_{6}$ and the edges in the images.

The algorithm for constructing the hierarchical parse graph is similar to the DDMCMC algorithm [39], [33] except that we adopt a deterministic strategy in this paper in generating the candidates and accepting the proposal. As the acceptance is not reversible, it is likely to get locally optimal solutions.

\section{EXPERIMENTS}

We test our algorithm on a number of scenes with rectangle structures and show both qualitative results through image reconstruction (or synthesis) by using the generative model and quantitative results through an ROC curve by comparing the performance of two approaches: 1) pure bottom-up rectangle detection and 2) our methods:

Qualitative results. We show six results of the computed configurations and synthesized images in Figs. 10 and 11. In these two figures, the first row shows the input images, the second row shows the edge detection results, the third row shows the detected and grouped rectangles in the final configurations and missing rectangles compared with the ground truth (with true positives, false positives, and missing rectangles being shown in different line styles), and the fourth row shows the reconstructed images based on the rectangle results in the third row. We can see that the reconstructed images miss some structures. Then, we add the generic sketches (curves) in the edges and final reconstructions are shown in the last row.

The image reconstruction proceeds in the following way: First, for the sketchable parts, we reconstruct the image from the image primitives after fitting some parameters for the intensity profiles. For the remaining area $\Lambda_{\text {nsk }}$, we follow [9] and divide $\Lambda_{\text {nsk }}$ into homogeneous texture regions by $k$-means clustering and then synthesize each texture region by sampling the Julesz ensemble so that the synthesized image has histograms matching the observed histograms of filter responses. More specifically, we compute the histograms of the derivative filters within a local window (e.g., $7 \times 7$ pixels). For example, we use seven filters and seven bins are used for each histogram. Then, in total, we have a 49-dimensional feature vector at each pixel. We then cluster these feature vectors into different regions. 
$\mathrm{r}_{2}$

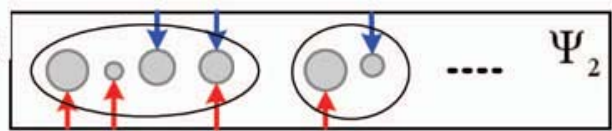

$\mathrm{r}_{3}$

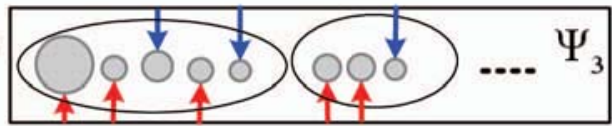

$\mathrm{r}_{4}$

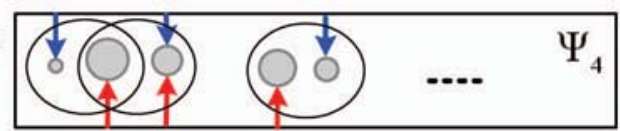

$\mathrm{r}_{6}$

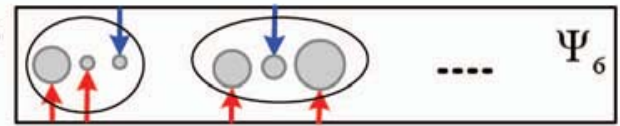

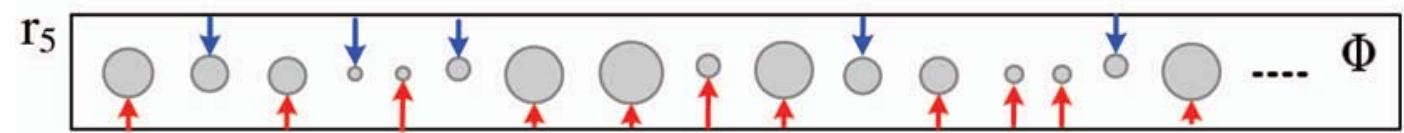

Fig. 8. Four sets of proposed candidates $\Psi_{2}, \Psi_{3}, \Psi_{4}$, and $\Psi_{6}$ for production rules $r_{2}, r_{3}, r_{4}$, and $r_{6}$, respectively, and the candidate set $\Phi$ for the instantiation rule $r_{5}$. Each circle represents a rectangle $\pi$ or a bounding box of a nonterminal node, while the associated upward (or downward) arrow indicates that it is generated through a bottom-up (or top-down) process. The size of the circle represents its weight $\omega(\pi)$. Each ellipse in $\Psi_{2}$, $\Psi_{3}, \Psi_{4}$, and $\Psi_{6}$ stands for a rectangle group candidate $\Pi$ which consists of a few circles. A circle may participate in more than one rectangle group candidate.

(a)

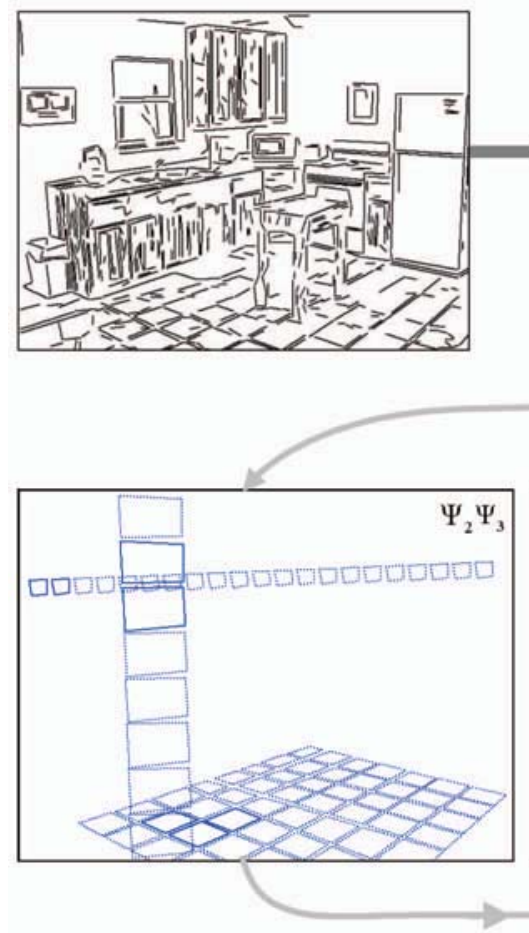

(d) (b)

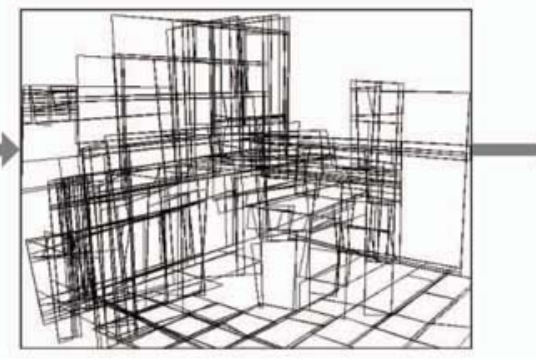

(c)

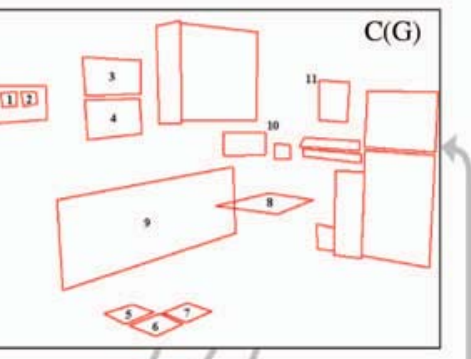

(e)

(f)

Fig. 9. A kitchen scene as running example. (a) Edge map. (b) Subset of $\Phi$ for rectangle candidates detected in phase 1. We show a subset for clarity. (c) Configuration $C(\mathbf{G})$ with a number of accepted rectangles in phase 2. (d)-(f) Candidates in $\Psi_{2}, \Psi_{3}, \Psi_{4}$, and $\Psi_{6}$, respectively. They are proposed based on the current node in $\mathbf{G}$ (i.e., shown in (b)).

In the computed configurations, some rectangles are missing due to the strong occlusion. For instance, some rectangles on the floor in the kitchen scene are missing due to the occlusion caused by the table on the floor. In addition, the results clearly show that high-level knowledge introduced by the graph grammar greatly improves the results. For example, in the building scene in the third column in Fig. 10, the windows become very weak on the left side of the image. By grouping them into a line rectangle group, the algorithm can recover these weak windows, which will not appear when using the likelihood model alone.

During our experiments, phase 1 is the most timeconsuming stage and takes about 2 minutes on a $640 \times 480$ image since we have to test many combinations to generate all of the rectangle proposals and build up their occlusion relations. Phases 2 and 3 are very fast and take about 1 minute altogether.

Quantitative evaluation. To evaluate our algorithm in a quantitative way, we collect a data set with 40 images. The average number of rectangles in each image of this data set is 38. Six have been shown in Figs. 10 and 11. We then manually annotate these images to get the ground truth for all the rectangles in each image.

Then, we randomly select 15 images from this data set as training data to tune all of the parameters and thresholds in our algorithm. After that, we run phase 2 and then phase 3 of 
(a)

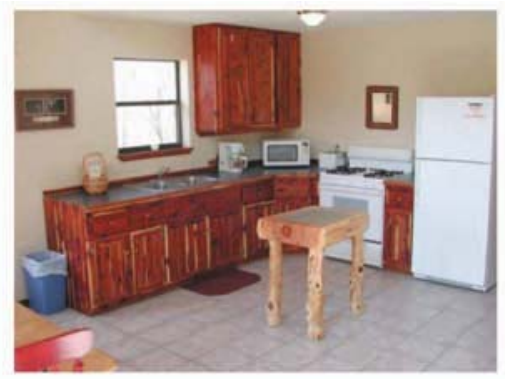

(b)

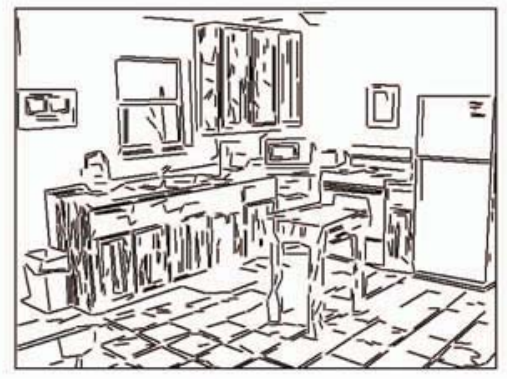

(c)

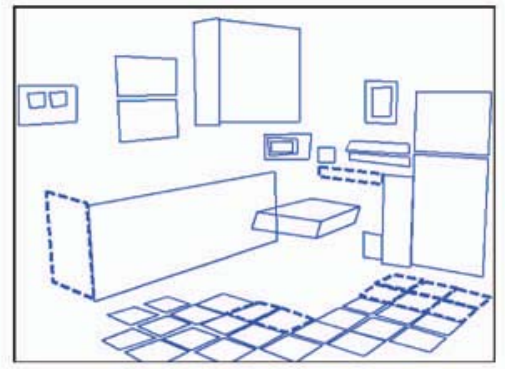

(d)

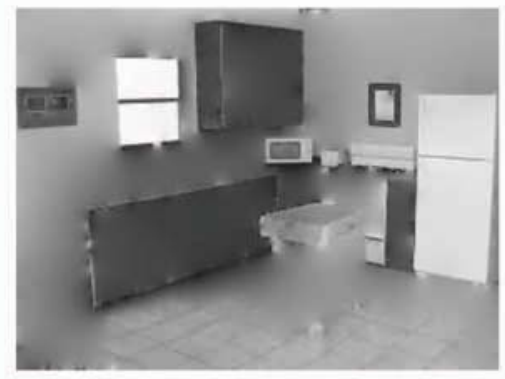

(e)

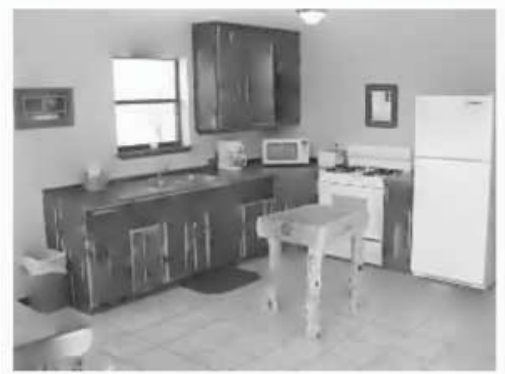

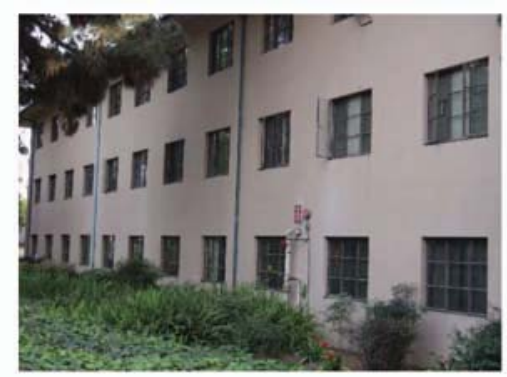
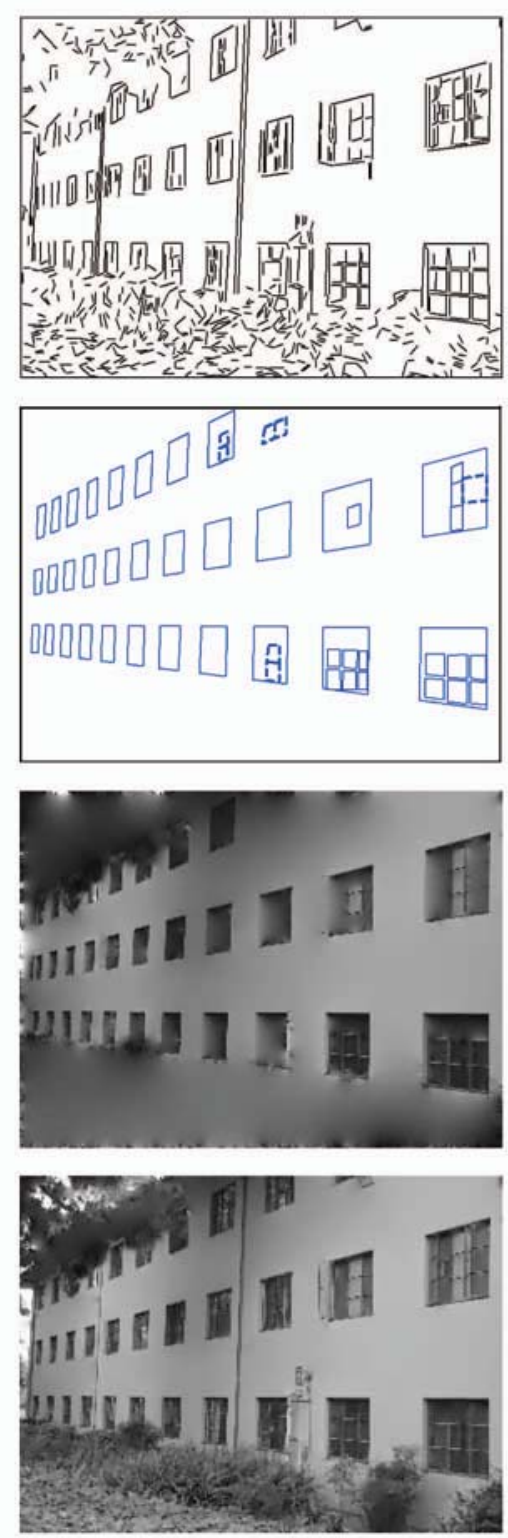
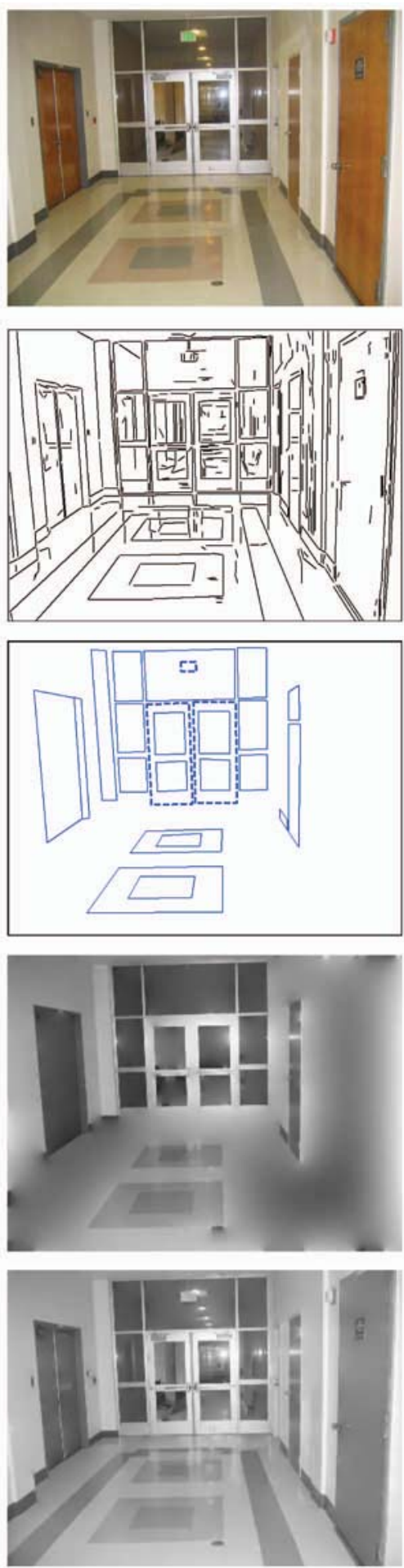

Fig. 10. Some experimental results. (a) Input image. (b) Edge map. (c) Computed rectangle configurations and missing rectangles compared with the ground truth: True positive rectangles are shown with solid lines, false positive rectangles are shown in dotted lines, and missing rectangles are shown in dashed lines. (d) Reconstructed image from the primal sketch model using the rectangle configurations only. (e) Reconstructed images after adding some background sketches to the configurations.

our algorithm on the rest images to generate detection results (note that the detection results shown in Figs. 10 and 11 are obtained when these six images are in testing data). Due to inherent randomness in splitting the data set into training data and testing data, we repeat the experiment six times. Fig. 12 shows the ROC curves with confidence intervals [23] for phase 2 (using bottom up only) and phase 3 (using both bottom up and top down), which are obtained by changing the threshold in phase 2. From these ROC curves, we can clearly see the dramatic improvement by using a topdown mechanism over the traditionally bottom-up mechanism only. Intuitively, some rectangles are nearly impossible to detect using the bottom-up methods and 
(a)

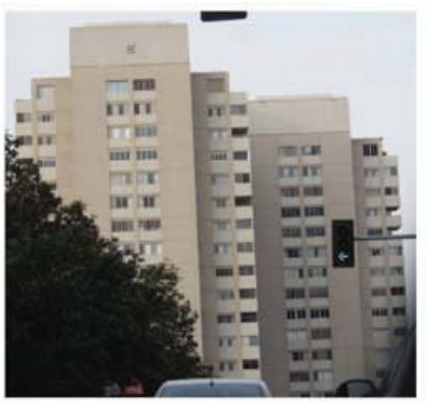

(b)

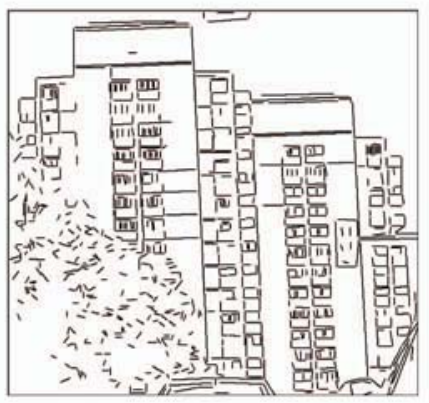

(c)

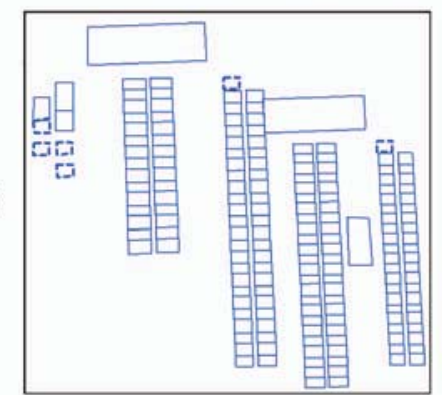

(d)

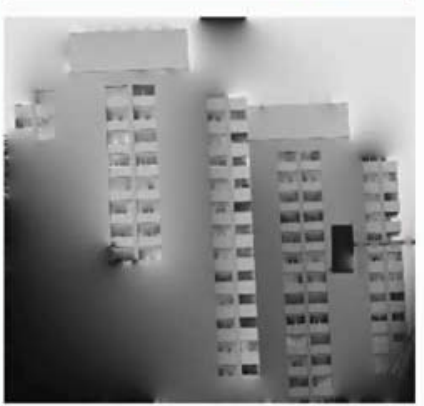

(e)

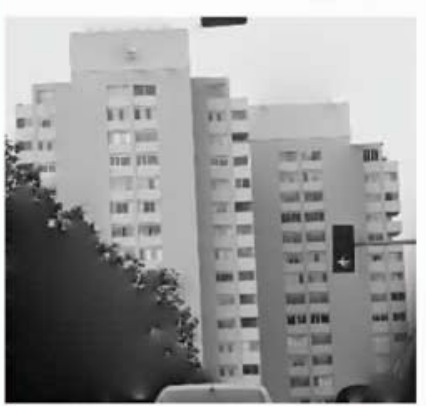

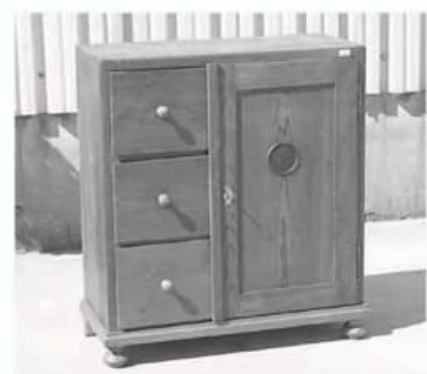
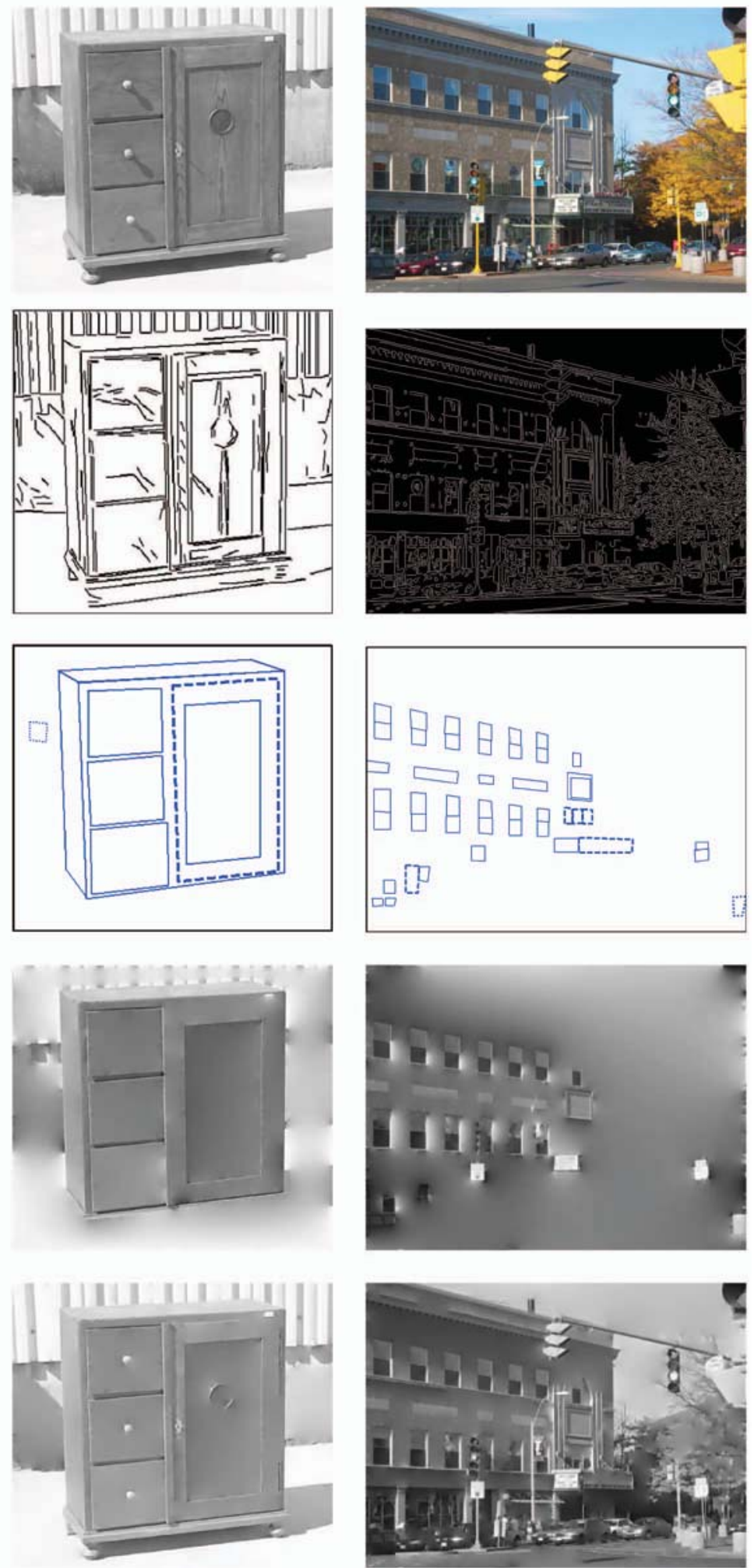

Fig. 11. More experimental results. (a) Input image. (b) Edge map. (c) Computed rectangle configurations and missing rectangles compared with the ground truth: True positive rectangles are shown with solid lines, false positive rectangles are shown in dotted lines, and missing rectangles are shown in dashed lines. (d) Reconstructed image from the primal sketch model using the rectangle configurations only. (e) Reconstructed images after adding some background sketches to the configurations.

can only be recovered through the context information using the grammar rules.

To plot the ROC curves, we need to classify each detected rectangle as either a true positive or a false alarm in comparison to a rectangle in the ground truth. To be considered as a correct detection, the area of overlap between the detected rectangle $a_{\operatorname{det}}$ and the ground truth rectangle $a_{\mathrm{gt}}$ is required to exceed 95 percent: 


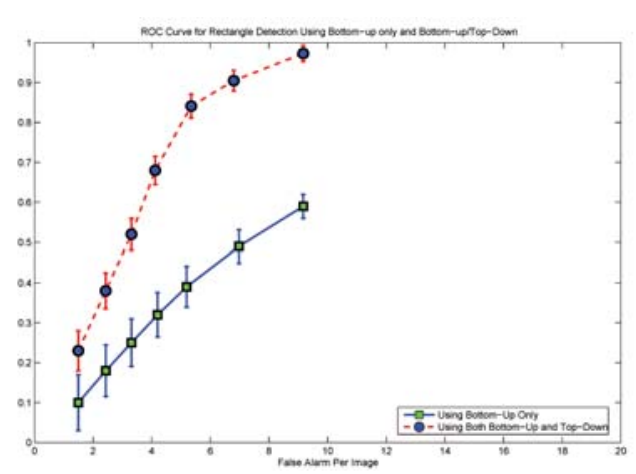

Fig. 12. ROC curves for the rectangle detection results by using bottom up only and using both bottom up and top down.

$$
\gamma=\frac{\operatorname{Area}\left(a_{\mathrm{det}} \cap a_{\mathrm{gt}}\right)}{\operatorname{Area}\left(a_{\mathrm{det}} \cup a_{\mathrm{gt}}\right)}>0.95 \text {. }
$$

\section{Discussion}

In this paper, we study an attribute grammar for image parsing in man-made scenes. This paper makes two main contributions to the vision literature. First, it uses an attributed grammar for incorporating prior knowledge. Such grammar representations have long been desired for high-level vision, especially scene understanding and parsing. Second, it integrates a top-down/bottom-up procedure for computing the parse graph with grammars. It extends the previous DDMCMC image parsing work [33] by including more flexible and hierarchical representations. The computing algorithm is compatible with the DDMCMC scheme, but we use deterministic ordering for efficiency considerations.

For our future work, we shall study the following aspects: 1) the image parsing is only for generic image interpretation in the current work. In ongoing projects, we are extending this framework to recognizing object categories [42], especially functional objects where objects within each category exhibit a wide range of structural variabilities [3]. The extended grammar will have many more production rules. 2) In the current work, we manually tune some probabilities and parameters in the energy function. These parameters should be learned automatically when we have a large number of manually parsed training examples, e.g., through supervised learning. We are currently collecting a large manually parsed image data set for learning grammars. An automatic learning algorithm is presented in a recent work [28], [42]. In experiments, we observe that the stopping thresholds $\Delta_{0}$ and $\Delta_{1}$ in phases 2 and 3 have to be decided by minimizing the detection errors (missing rate and false alarm) and cannot be decided by the posterior probability alone.

\section{ACKNOWLEDGMENTS}

This work was supported in part by a subaward of the W.M. Keck Foundation, the Chinese 863 Grant 2006AA01Z121, the US National Science Foundation under Grant IIS-0413214 and grant IIS-0713652, and the US Office of Naval Research under Grant N00014-05-01-0543. The authors would like to thank the Lotus Hill Institute for its assistance in preparing the data set and the Lotus Hill Data Annotation [44]. They would also like to thank the anonymous reviewers, whose constructive comments helped improve this paper in many aspects

\section{RefERENCES}

[1] S. Baumann, "A Simplified Attribute Graph Grammar for HighLevel Music Recognition," Proc. Third Int'l Conf. Document Analysis and Recognition, 1995.

[2] R. Brooks, "Symbolic Reasoning Among 3D Models and 2D Images," Stanford AIM-343, STAN-CS-81-861, 1981.

[3] H. Chen, Z.J. Xu, and S.C. Zhu, "Composite Templates for Cloth Modeling and Sketching," Proc. IEEE Int'l Conf. Computer Vision and Pattern Recognition, June 2006.

[4] A.R. Dick, P.H.S. Torr, and R. Cipolla, "Modeling and Interpretation of Architecture from Several Images," Int'l J. Computer Vision, vol. 60, no. 2, pp. 111-134, 2004.

[5] S. Dickinson, A. Pentland, and A. Rosenfeld, "3-D Shape Recovery Using Distributed Aspect Matching," IEEE Trans. Pattern Analysis and Machine Intelligence, vol. 14, no. 2, pp. 174-198, Feb. 1992.

[6] T. Fan, G. Medioni, and R. Nevatia, "Recognizing 3-D Objects Using Surface Descriptions," IEEE Trans. Pattern Analysis and Machine Intelligence, vol. 11, no. 11, pp. 1140-1157, Nov. 1989.

[7] M.A. Fischler and R.C. Bolles, "Random Sample Consensus: A Paradigm for Model Fitting with Applications to Image Analysis and Automated Cartography," Comm. ACM, vol. 24, pp. 381-395, 1981.

[8] K.S. Fu, Syntactic Pattern Recognition and Applications. Prentice Hall, 1981.

[9] C.E. Guo, S.C. Zhu, and Y.N. Wu, "A Mathematical Theory of Primal Sketch and Sketchability," Proc. Ninth IEEE Int'l Conf. Computer Vision, 2003.

[10] C.E. Guo, S.C. Zhu, and Y.N. Wu, "Primal Sketch: Integrating Texture and Structure," Computer Vision and Image Understanding, vol. 106, no. 1, pp. 5-19, Apr. 2007.

[11] A. Hanson and E. Riseman, "Visions: A Computer System for Interpreting Scenes," Computer Vision Systems, 1978.

[12] K. Huang, W. Hong, and Y. Ma, "Symmetry-Based Photo Editing," Proc. First IEEE Workshop Higher-Level Knowledge in 3D Modeling \& Motion Analysis, 2003.

[13] V. Hwang and T. Matsuyama, "SIGMA: A Framework for Image Understanding: Integration of Bottom-Up and Top-Down Analyses," Proc. Int'l Joint Conf. Artificial Intelligence '85, pp. 908-915, 1985

[14] V. Hwang, L.S. Davis, and T. Matsuyama, "Hypothesis Integration in Image Understanding Systems," Computer Vision, Graphics, and Image Processing, vol. 36, nos. 2/3, pp. 321-371, 1986.

[15] S. Ioffe and D. Forsyth, "Probabilistic Methods for Finding People," Int'l J. Computer Vision, vol. 43, no. 1, pp. 45-68, 2001.

[16] Y. Jin and S. Geman, "Context and Hierarchy in a Probabilistic Image Model," Proc. IEEE Int'l Conf. Computer Vision and Pattern Recognition, June 2006.

[17] D. Lagunovsky and S. Ablameyko, "Straight-Line-Based Primitive Extraction in Grey-Scale Object Recognition," Pattern Recognition Letters, vol. 20, no. 10, pp. 1005-1014, Oct. 1999.

[18] A. Levinshtein, C. Sminchisescu, and S.J. Dickinson, "Learning Hierarchical Shape Models from Examples," Proc. Fifth Int'l Workshop Energy Minimization Methods in Computer Vision and Pattern Recognition, 2005.

[19] C. Lin and R. Nevatia, "Building Detection and Description from a Single Intensity Image," Computer Vision and Image Understanding, vol. 72, no. 2, pp. 101-121, 1998.

[20] S. Mallat and Z. Zhang, "Matching Pursuit with Time-Frequency Dictionaries," IEEE Trans. Signal Processing, vol. 41, no. 12, pp. 3397-3415, 1993.

[21] W. Mann and T. Binford, "Successor: Interpretation Overview and Constraint System," Proc. Image Understanding Workshop, pp. 15051518, 1996.

[22] D. McKeown, W. Harvey, and L. Wixson, "Automating Knowledge Acquisition for Aerial Image Interpretation," Computer Vision, Graphics, and Image Processing, vol. 46, no. 1, pp. 37-81, 1989.

[23] S. Munder and D. Gavrila, "An Experimental Study on Pedestrian Classification," IEEE Trans. Pattern Analysis and Machine Intelligence, vol. 28, no. 11, Nov. 2006. 
[24] Y. Ohta, T. Kanade, and T. Sakai, "An Analysis System for Scenes Containing Objects with Substructures," Proc. Fourth Int'l Conf. Pattern Recognition, pp. 752-754, 1978.

[25] Y. Ohta, Knowledge-Based Interpretation of Outdoor Natural Color Scenes. Pitman, 1985.

[26] I. Pollak, J.M. Siskind, M.P. Harper, and C.A. Bouman, "Parameter Estimation for Spatial Random Trees Using the EM Algorithm," Proc. IEEE Int'l Conf. Image Processing, 2003.

[27] I. Pollak, J.M. Siskind, M.P. Harper, and C.A. Bouman, "Modeling and Estimation of Spatial Random Trees with Application to Image Classification," Proc. 28th IEEE Int'l Conf. Acoustics, Speech, and Signal Processing, 2003.

[28] J. Porway, B. Yao, and S.C. Zhu, "Learning Compositional Models for Object Categories from Small Sample Sets," Object Categorization: Computer and Human Vision Perspectives, S. Dickinson et al., eds., Cambridge Univ. Press, 2009.

[29] J. Rekers and A. Schürr, "Defining and Parsing Visual Languages with Layered Graph Grammars," J. Visual Language and Computing, Sept. 1996.

[30] K. Siddiqi, A. Shokoufandeh, S.J. Dickinson, and S.W. Zucker, "Shock Graphs and Shape Matching," Int'l J. Computer Vision, vol. 35, no. 1, pp. 13-32, 1999.

[31] J.M. Siskind, J. Sherman, I. Pollak, M.P. Harper, and C.A. Bouman, "Spatial Random Tree Grammars for Modeling Hierarchal Structure in Images," IEEE Trans. Pattern Analysis and Machine Intelligence, vol. 29, no. 9, pp. 1504-1519, Sept. 2007.

[32] W.-B. Tao, J.-W. Tian, and J. Liu, "A New Approach to Extract Rectangle Building from Aerial Urban Images," Proc. Sixth Int'l Conf. Signal Processing, pp. 143-146, 2002.

[33] Z.W. Tu, X.R. Chen, A.L. Yuille, and S.C. Zhu, "Image Parsing: Unifying Segmentation, Detection and Recognition," Int'l J. Computer Vision, vol. 63, no. 2, pp. 113-140, 2005.

[34] W. Wang, I. Pollak, T.-S. Wong, C.A. Bouman, M.P. Harper, and J.M. Siskind, "Hierarchical Stochastic Image Grammars for Classification and Segmentation," IEEE Trans. Image Processing, vol. 15 , no. 10 , pp. 3033-3052, Oct. 2006.

[35] T.F. Wu, G.S. Xia, and S.C. Zhu, "Compositional Boosting for Computing Hierarchical Image Structures," Proc. IEEE. Int'l Conf. Computer Vision and Pattern Recognition, June 2007.

[36] F.C You and K.S. Fu, "A Syntactic Approach to Shape Recognition Using Attributed Grammars," IEEE Trans. Systems, Man, and Cybernetics, vol. 9, pp. 334-345, 1979.

[37] F.C. You and K.S. Fu, "Attributed Grammar: A Tool for Combining Syntactic and Statistical Approaches to Pattern Recognition," IEEE Trans. Systems, Man, and Cybernetics, vol. 10, pp. 873-885, 1980.

[38] W. Zhang and J. Kosecka, "Extraction, Matching and Pose Recovery Based on Dominant Rectangular Structures," Proc. First IEEE Workshop Higher-Level Knowledge in 3D Modeling and Motion Analysis, 2003.

[39] S.C. Zhu, R. Zhang, and Z.W. Tu, "Integrating Top-Down/ Bottom-Up for Object Recognition by DDMCMC," Proc. IEEE Int'l Conf. Computer Vision and Pattern Recognition, 2000.

[40] S.C. Zhu and A.L. Yuille, "FORMS: A Flexible Object Recognition and Modeling System," Int'l J. Computer Vision, vol. 20, no. 3, pp. 187-212, 1996.

[41] S.C. Zhu, Y.N. Wu, and D. Mumford, "Minimax Entropy Principle and Its Application to Texture Modeling," Neural Computation, vol. 9, pp. 1627-1660, 1997.

[42] S.C. Zhu and D. Mumford, "A Stochastic Grammar of Images," Foundations and Trends in Computer Graphics and Vision, vol. 2, no. 4, pp. 259-362, 2006.

[43] Y. Zhu, B. Carragher, F. Mouche, and C. Potter, "Automatic Particle Detection through Efficient Hough Transforms," IEEE Trans. Medical Imaging, vol. 22, no. 9, pp. 1053-1062, Sept. 2003.

[44] Z.Y. Yao, X. Yang, and S.C. Zhu, "Introduction to a Large Scale General Purpose Groundtruth Dataset: Methodology, Annotation Tool, and Benchmarks," Proc. Sixth Int'l Conf. EMMCVPR, Aug. 2007.

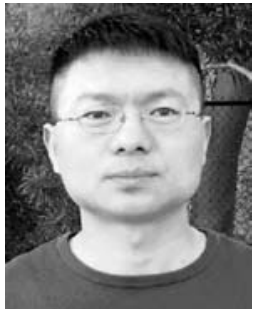

Feng Han received the BS degree in computer science from Dalian University of Technology in 1996, the ME degree in electrical and electronic engineering from Nanyang Technological University in 2000, and the $\mathrm{PhD}$ degree in computer science from the University of California, Los Angeles (UCLA) in 2005. He is currently a member of the technical staff in the Vision and Learning Laboratory at Sarnoff Corp. His research interests include computer vision, image processing, pattern recognition, and computer graphics.

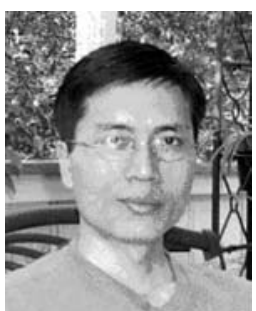

Song-Chun Zhu received the BS degree from the University of Science and Technology of China in 1991 and the MS and PhD degrees from Harvard University in 1994 and 1996, respectively. He is currently a professor with the Department of Statistics and the Department of Computer Science at the University of California, Los Angeles (UCLA). Before joining UCLA, he was a postdoctoral researcher in the Division of Applied Math at Brown University from 1996 to 1997, a lecturer in the Department of Computer Science at Stanford University from 1997 to 1998, and an assistant professor of computer science at Ohio State University from 1998 to 2002. His research interests include computer vision and learning, statistical modeling, and stochastic computing. He has published more than 100 papers in computer vision. He has received a number of honors, including the David Marr Prize in 2003, the J.K. Aggarwal prize in 2008, the Marr Prize honorary nominations in 1999 and 2007, a Sloan Fellowship in Computer Science in 2001, a US National Science Foundation Early Career Development Award in 2001, and an US Office of Naval Research Young Investigator Award in 2001. In 2005, he founded, with friends, the Lotus Hill Institute for Computer Vision and Information Science in China as a nonprofit research organization (www.lotushill.org).

$\triangleright$ For more information on this or any other computing topic, please visit our Digital Library at www.computer.org/publications/dlib. 